\title{
q-TITCHMARSH-WEYL THEORY: SERIES EXPANSION
}

\author{
M. H. ANNABY, Z. S. MANSOUR, AND I. A. SOLIMAN
}

\begin{abstract}
We establish a $q$-Titchmarsh-Weyl theory for singular $q$-SturmLiouville problems. We define $q$-limit-point and $q$-limit circle singularities, and we give sufficient conditions which guarantee that the singular point is in a limit-point case. The resolvent is constructed in terms of Green's function of the problem. We derive the eigenfunction expansion in its series form. A detailed worked example involving Jackson $q$-Bessel functions is given. This example leads to the completeness of a wide class of $q$-cylindrical functions.
\end{abstract}

\section{$\S 1$. Introduction and preliminaries}

The work in singular Sturm-Liouville problems of the type

$$
\begin{gathered}
-y^{\prime \prime}+\nu(x) y=\lambda y, \quad 0 \leq x<\infty \\
\cos \alpha y(0)+\sin \alpha y^{\prime}(0)=0
\end{gathered}
$$

was established in the works of Weyl ([48]-[50]), although, according to Titchmarsh [47], it may go back to Sturm and Liouville. Here $\nu(\cdot) \in C[0, \infty)$, $\alpha \in[0, \pi)$, and $\lambda \in \mathbb{C}$ is the eigenvalue parameter. Several problems are associated with (1.1) and (1.2). First is the limit-point, limit-circle classification, which goes back to Weyl [50]. The eigenfunction expansion theorem has been tackled in different ways independently by many authors and using different techniques. The first study of the eigenfunction expansion problem is by Weyl [50] using Fredholm's theory of integral equations. Operator theoretic approaches could be found in the works of Stone ([40], [41]), Naimark [39], and Berezanskii [13]. Titchmarsh used function theory techniques as in [47]. The problem is also investigated using other techniques by Yosida ([51], [52]), Levinson [35], and Levitan and Sargsjan ([36], [37]).

Received May 22, 2010. Revised June 1, 2011. Accepted August 9, 2011.

2010 Mathematics Subject Classification. 34B24, 34L10, 39A13.

Mansour's work was partially supported by King Saud University grant Distinguished Scientist Fellowship Program/MATH 01.

(C) 2012 by The Editorial Board of the Nagoya Mathematical Journal 
Our goal is to establish a study of singular Sturm-Liouville $q$-difference operators when the derivative in (1.1) and (1.2) is replaced by Jackson's qdifference operator $D_{q}$ (definitions are given below). We follow Titchmarsh's technique (see [47, Chapter 2]). We define the limit-point, limit-circle cases in the $q$-setting and give a classification criterion. We construct Green's function and derive eigenfunction expansion theorems. We introduce detailed worked examples involving $q$-Bessel eigenvalue problems. This leads us to the completeness of several families of $q$-Bessel and cylindrical functions. We would like to mention here that the completeness and asymptotics of families of the third-type $q$-Bessel functions have received lots of attention in many recent studies (see [1]-[4], [8], [10], [11], [31], [32], [45]). In many interesting examples, the potential $\nu(\cdot)$ has singularities at one or both endpoints of the interval $[0, a]$, or the interval is extended to infinity. In this paper we discuss in detail the case of the infinite interval $(0, \infty), \nu$ as continuous at zero. The case of a finite interval with singularity at one endpoint or both is basically similar. The proofs concerning the expansion theorems are established by means of contour integration and the calculus of residues. This technique was established by Cauchy and was implemented by Weyl [48] and Titchmarsh [46] to prove the expansions of the singular classical Sturm-Liouville problem (see [46], [47], [50]). It is worthwhile to mention that the problem of expendability of functions in terms of $q$-orthogonal functions, which seems to be first discussed by Carmichael ([18]-[20]), has attracted the work of several authors (see, e.g., [16], [17], [28], [42], [43]). Due to the speciality of the $q$-theory, general results from the operator theory (see, e.g., [13]) might not be directly applicable. In fact, as is seen below, even direct investigations need the derivations of $q$-analogs of several classical results. Also, the application of other approaches like those of [36] and [37] needs special investigation of $q$-analogs of classical analysis concepts and results, like the $q$-analog of functions of bounded variation and related results.

Now we introduce some of the $q$-notations and $q$-results which will be used throughout the paper. Hereafter, $q \in(0,1)$ is fixed. We use the standard notations found in [7] and [27]. A set $A \subseteq \mathbb{R}$ is called q-geometric if, for every $x \in A, q x \in A$. Let $f$ be a real- or complex-valued function defined on a $q$-geometric set $A$. The $q$-difference operator is defined by

$$
D_{q} f(x):=\frac{f(x)-f(q x)}{x(1-q)}, \quad x \neq 0 .
$$


If $0 \in A$, the $q$-derivative at zero is defined to be

$$
D_{q} f(0):=\lim _{n \rightarrow \infty} \frac{f\left(x q^{n}\right)-f(0)}{x q^{n}}, \quad x \in A,
$$

if the limit exists and does not depend on $x$. Since the formulation of selfadjoint eigenvalue problems requires $D_{q^{-1}}$, we define it for $x \in A$ to be

$$
D_{q^{-1}} f(x):= \begin{cases}\frac{f(x)-f\left(q^{-1} x\right)}{x\left(1-q^{-1}\right)}, & x \neq 0, \\ D_{q} f(0), & x=0,\end{cases}
$$

provided that $D_{q} f(0)$ exists. A right inverse, $q$-integration of the $q$-difference operator $D_{q}$ is defined by Jackson [30] as

$$
\int_{0}^{x} f(t) d_{q} t:=x(1-q) \sum_{n=0}^{\infty} q^{n} f\left(x q^{n}\right), \quad x \in A,
$$

provided that the series converges. In general,

$$
\int_{a}^{b} f(t) d_{q} t:=\int_{0}^{b} f(t) d_{q} t-\int_{0}^{a} f(t) d_{q} t, \quad a, b \in A .
$$

There is no unique canonical choice for the $q$-integration over $[0, \infty)$. Hahn [24] defined the $q$-integration for a function $f$ over $[0, \infty)$ by

$$
\int_{0}^{\infty} f(t) d_{q} t=(1-q) \sum_{n=-\infty}^{\infty} q^{n} f\left(q^{n}\right),
$$

while Matsuo [38] defined $q$-integration on the interval $[0, \infty)$ by

$$
\int_{0}^{\infty} f(t) d_{q} t:=b(1-q) \sum_{n=-\infty}^{\infty} q^{n} f\left(b q^{n}\right), \quad b>0,
$$

provided that the series converges. From now on, we will only deal with Hahn $q$-integration on $(0, \infty)$. A $q$-analog of the fundamental theorem of calculus is given by

$$
D_{q} \int_{0}^{x} f(t) d_{q} t=f(x), \quad \int_{0}^{x} D_{q} f(t) d_{q} t=f(x)-\lim _{n \rightarrow \infty} f\left(x q^{n}\right),
$$

where $\lim _{n \rightarrow \infty} f\left(x q^{n}\right)$ can be replaced by $f(0)$ if $f$ is $q$-regular at zero, that is, if

$$
\lim _{n \rightarrow \infty} f\left(x q^{n}\right)=f(0), \quad \text { for all } x \in A
$$


Corollary 1.1. Let $f$ be a function defined on a q-geometric set $A$. If $x \in q A:=\{q t: t \in A\}$, then

$$
\begin{aligned}
& D_{q^{-1}} \int_{0}^{x} f(t) d_{q} t=q f\left(q^{-1} x\right) \\
& \int_{0}^{x} D_{q^{-1}} f(t) d_{q} t=q f\left(q^{-1} x\right)-q \lim _{n \rightarrow \infty} f\left(x q^{n-1}\right) .
\end{aligned}
$$

Proof. For any $x \in q A$, we have

$$
D_{q^{-1}} f(x)=D_{q, q^{-1} x} f\left(q^{-1} x\right)=q D_{q, x} f\left(q^{-1} x\right) .
$$

Then, applying (1.6), we obtain

$$
D_{q^{-1}} \int_{0}^{x} f(t) d_{q} t=q D_{q, x} \int_{0}^{q^{-1} x} f(t) d_{q} t=q f\left(q^{-1} x\right)
$$

and

$$
\int_{0}^{x} D_{q^{-1}} f(t) d_{q} t=q \int_{0}^{x} D_{q, x} f\left(q^{-1} t\right) d_{q} t=q f\left(q^{-1} x\right)-q \lim _{n \rightarrow \infty} f\left(x q^{n-1}\right) .
$$

The $q$-type product formula which we apply in this paper is the $q$-Leibnitz formula,

$$
D_{q}(f g)(x)=g(x) D_{q} f(x)+f(q x) D_{q} g(x),
$$

and hence the $q$-integration by parts is given by

$$
\int_{0}^{a} g(x) D_{q} f(x) d_{q} x=(f g)(a)-\lim _{n \rightarrow \infty}(f g)\left(x q^{n}\right)-\int_{0}^{a} f(q x) D_{q} g(x) d_{q} x .
$$

The symmetric Leibnitz formula is

$$
D_{q}(f g)(x)=g(x) D_{q} f(x)+f(x) D_{q} g(x)-x(1-q) D_{q} f(x) D_{q} g(x) .
$$

If $f, g$ are $q$-regular at zero, then $\lim _{n \rightarrow \infty}(f g)\left(x q^{n}\right)$ in (1.8) will be replaced by $(f g)(0)$. If $g(x) \neq 0$ for all $x \in A$, then the $q$-derivative of the quotient $f / g$ is given by

$$
D_{q}\left(\frac{f}{g}\right)=\frac{g(x) D_{q} f(x)-f(x) D_{q} g(x)}{g(x) g(q x)}, \quad x \in A .
$$


Let $L_{q}^{2}(0, a)$ be the space of all complex-valued functions defined on $[0, a]$ such that

$$
\int_{0}^{a}|f(t)|^{2} d_{q} t<\infty
$$

The space $L_{q}^{2}(0, a)$ associated with the inner product

$$
\langle f, g\rangle_{0}=\int_{0}^{a} f(t) \overline{g(t)} d_{q} t, \quad f, g \in L_{q}^{2}(0, a)
$$

is a separable Hilbert space (see [8]). Let $L_{q}^{2}(0, \infty)$ be the space of all functions $f$ defined on $[0, \infty)$ for which

$$
\int_{0}^{\infty}|f(t)|^{2} d_{q} t<\infty
$$

Let $\mathcal{H}:=L_{q}^{2}((0, \infty),\langle\cdot, \cdot\rangle)$ be the space $L_{q}^{2}(0, \infty)$ associated with the inner product $\langle\cdot, \cdot\rangle$ defined by

$$
\langle f, g\rangle:=\int_{0}^{\infty} f(x) \overline{g(x)} d_{q} x, \quad f, g \in L_{q}^{2}(0, \infty) .
$$

If $f \in \mathcal{H}$, then $f \in L_{q}^{2}\left(0, q^{-m}\right)$ for all $m \in \mathbb{N}$.

Lemma 1.2. Let $(X,\langle\cdot, \cdot\rangle)$ be an inner product space over $\mathbb{C}$. Then for any $x, y$ in $X$ and $\alpha \in \mathbb{C}$, we have

$$
\|x+\alpha y\|^{2} \geq \frac{1}{2}|\alpha|^{2}\|y\|^{2}-\|x\|^{2} .
$$

Proof. Since

$$
\left(\|x\|-\frac{|\alpha|}{2}\|y\|\right)^{2} \geq 0, \quad|\Re \bar{\alpha}\langle x, y\rangle| \leq|\alpha|\|x\|\|y\|,
$$

then

$$
\Re \bar{\alpha}\langle x, y\rangle \geq-|\alpha|\|x\|\|y\| \geq-\|x\|^{2}-\frac{|\alpha|^{2}}{4}\|y\|^{2}
$$

But

$$
\|x+\alpha y\|^{2}=\|x\|^{2}+2 \Re \bar{\alpha}\langle x, y\rangle+|\alpha|^{2}\|y\|^{2} .
$$

Substituting from (1.12) into (1.13) implies (1.11). 
In [9], the regular $q$-Sturm-Liouville problem

$$
\begin{aligned}
-\frac{1}{q} D_{q^{-1}} D_{q} y(x)+u(x) y(x) & =\lambda y(x), \\
U_{1}(y) & :=a_{11} y(0)+a_{12} D_{q^{-1}} y(0)=0, \\
U_{2}(y) & :=a_{21} y(a)+a_{22} D_{q^{-1}} y(a)=0
\end{aligned}
$$

is studied where $0 \leq x \leq a<\infty, \lambda \in \mathbb{C}$, and $u(\cdot)$ is a continuous at zero realvalued function. The numbers $\left\{a_{i j}\right\}, i, j \in\{1,2\}$ are arbitrary real numbers such that the rank of the matrix $\left(a_{i j}\right)_{1 \leq i, j \leq 2}$ is 2 . It is proved that problem (1.14)-(1.16) has a denumerable set of real and simple eigenvalues $\left\{\lambda_{n}\right\}_{n=0}^{\infty}$ and that the set of corresponding normalized eigenfunctions, $\left\{\psi_{n}(\cdot)\right\}_{n=0}^{\infty}$, is an orthonormal basis in the Hilbert space $L_{q}^{2}(0, a)$. The theory of regular $q$-Sturm-Liouville problems has been applied in quantum mechanical problems (see, e.g., [34]). It is expected that the theory developed in the present paper will be applied in more $q$-models since, as far as we know, it is the first treatment of the half-line theory. A spectral theory for $q$-Sturm-Liouville problems based on the Askey-Wilson operator is still under investigation. There are few publications in that direction. (For some solutions and applications on $q$-Sturm-Liouville problems of Askey-Wilson operators, see [14], [15], [43], [21].)

\section{$\S 2$. Fundamental solutions}

In this section we investigate the properties of fundamental solutions of the second-order $q$-difference equation (1.14) on $x \in[0, \infty)$. The existence of a fundamental set of solutions of (1.14) was proved in [9] on a finite interval of the form $[0, a], 0<a<\infty$. One can see that the proof is similar when the finite interval is extended to the half-line $[0, \infty)$. So we introduce the following theorem without proof. We first define the class $C_{q}^{2}(0, \infty)$ to be the set of all functions $y$ defined on $\left[0, \infty\left[\right.\right.$ such that $y(\cdot)$ and $D_{q} y(\cdot)$ are continuous at zero. Recall that continuity at zero implies $q$-regularity at zero, but the converse is not necessarily true.

THEOREM 2.1. For $c_{1}, c_{2} \in \mathbb{C}$, equation (1.14) has a unique solution $y(\cdot, \lambda) \in C_{q}^{2}(0, \infty)$ which satisfies

$$
y(0, \lambda)=c_{1}, \quad D_{q^{-1}} y(0, \lambda)=c_{2}, \quad \lambda \in \mathbb{C} .
$$

Moreover, $y(x, \lambda)$ is entire in $\lambda$ for all $x \in[0, \infty)$. 
For convenience, let

$$
L_{x}:=-\frac{1}{q} D_{q^{-1}} D_{q}+u(x) .
$$

We also denote by $W_{q}(y, z)$ the $q$-Wronskian of any two solutions of equation (1.14); that is (see, e.g., [5], [45]),

$$
W_{q}(y, z)(\cdot):=y(\cdot) D_{q} z(\cdot)-z(\cdot) D_{q} y(\cdot) .
$$

REMARK 2.2. The function $y(\cdot, \lambda)$ satisfies the $q$-regularity condition

$$
\lim _{n \rightarrow \infty} y\left(x q^{n}, \lambda\right)=y(0, \lambda)
$$

uniformly on any compact subset of the $\lambda$ plane, for any $x>0$. This result is not proved in [9], so we outline its proof here. Indeed, the solution $y(\cdot, \lambda)$ is the uniform limit as $m \rightarrow \infty$ of the sequence of successive approximations

$$
\begin{aligned}
y_{1}(x, \lambda)= & c_{1} \varphi_{1}(x, \lambda)+c_{2} \varphi_{2}(x, \lambda), \\
y_{m+1}(x, \lambda)= & c_{1} \varphi_{1}(x, \lambda)+c_{2} \varphi_{2}(x, \lambda) \\
& -q \int_{0}^{x}\left\{\varphi_{2}(x, \lambda) \varphi_{1}(q t, \lambda)-\varphi_{1}(x, \lambda) \varphi_{2}(q t, \lambda)\right\} \\
& \times u(q t) y_{m}(q t, \lambda) d_{q} t,
\end{aligned}
$$

where $\varphi_{1}(\cdot, \lambda)$ and $\varphi_{1}(\cdot, \lambda)$ are, respectively, the $q$-cosine and $q$-sine functions

$$
\begin{aligned}
& \varphi_{1}(x, \lambda)=\cos (s x ; q):=\sum_{n=0}^{\infty}(-1)^{n} \frac{q^{n^{2}}(s x(1-q))^{2 n}}{(q ; q)_{2 n}}, \\
& \varphi_{2}(x, \lambda)= \begin{cases}\frac{\sin (s x ; q)}{s}:=s^{-1} \sum_{n=0}^{\infty}(-1)^{n} \frac{q^{n(n+1)}(x(1-q))^{2 n+1}}{(q ; q)_{2 n+1}}, & \lambda \neq 0 \\
x, & \lambda=0\end{cases}
\end{aligned}
$$

and $s:=\sqrt{\lambda}$ is defined to be the principal branch. The functions $\left\{\varphi_{i}(\cdot, \lambda)\right\}_{i=1}^{2}$ form a fundamental set of solutions for the equation

$$
\frac{1}{q} D_{q^{-1}} D_{q} y(x)+\lambda y(x)=0,
$$

with the $q$-Wronskian $W_{q}\left(\varphi_{1}(\cdot, \lambda), \varphi_{2}(\cdot, \lambda)\right) \equiv 1$. Using that $\lim _{n \rightarrow \infty} \varphi_{i}\left(x q^{n}\right.$, $\lambda)=\varphi_{i}(0, \lambda)$ uniformly on any compact subset of $\mathbb{C}, i=1,2$, one can prove that $y_{m}(\cdot, \lambda), m \in \mathbb{N}$, have the same regularity property. Then, so does $y(\cdot, \lambda)$ because it is the uniform limit of $y_{m}(\cdot, \lambda)$ on any closed subinterval $[0, a], a>$ 0 and any compact subset of $\mathbb{C}$. 
Let $\alpha \in \mathbb{R}$ be fixed, and let $\{\phi(\cdot, \lambda), \theta(\cdot, \lambda)\}$ be the fundamental set of solutions of (1.14) determined subject to the initial conditions

$$
\begin{aligned}
& \phi(0, \lambda)=\cos \alpha, \quad D_{q^{-1}} \phi(0, \lambda)=\sin \alpha, \\
& \theta(0, \lambda)=\sin \alpha, \quad D_{q^{-1}} \theta(0, \lambda)=-\cos \alpha .
\end{aligned}
$$

Lemma 2.3. For $x \in[0, \infty)$ and $\lambda \in \mathbb{C}$, we have

$$
\overline{\phi(x, \lambda)}=\phi(x, \bar{\lambda}), \quad \overline{\theta(x, \lambda)}=\theta(x, \bar{\lambda}) .
$$

Proof. It suffices to prove the lemma for $\phi(\cdot, \lambda)$. Since $\phi(\cdot, \lambda)$ is a solution of (1.14), then

$$
-\frac{1}{q} D_{q^{-1}} D_{q} \phi(x, \lambda)+u(x) \phi(x, \lambda)=\lambda \phi(x, \lambda), \quad x \in[0, \infty) .
$$

Taking the complex conjugate, we obtain

$$
-\frac{1}{q} D_{q^{-1}} D_{q} \overline{\phi(x, \lambda)}+u(x) \overline{\phi(x, \lambda)}=\bar{\lambda} \overline{\phi(x, \lambda)}, \quad x \in[0, \infty) .
$$

Then $\overline{\phi(x, \lambda)}$ is a solution of the equation

$$
-\frac{1}{q} D_{q^{-1}} D_{q} z(x)+u(x) z(x)=\bar{\lambda} z(x), \quad x \in[0, \infty)
$$

with the initial conditions (2.6). But $z=\phi(x, \bar{\lambda})$ is also a solution of $(2.8)$ with the same initial conditions (2.6). From the uniqueness of solutions, the lemma follows for $\phi(x, \lambda)$.

Lemma 2.4. For any two functions $y$ and $z$ in $C_{q}^{2}(0, \infty)$, we have Green's identity

$$
\int_{0}^{x}\left(y L_{t} z-z L_{t} y\right) d_{q} t=W_{q}(y, z)(0)-W_{q}(y, z)\left(x q^{-1}\right)
$$

for all $x \in(0, \infty)$.

Proof. From (2.2) and (1.7), we obtain for any $x>0$,

$$
\begin{aligned}
\int_{0}^{x} y L_{t} z-z L_{t} y d_{q} t & =\frac{-1}{q} \int_{0}^{x}\left(y(t) D_{q^{-1}} D_{q} z(t)-z(t) D_{q^{-1}} D_{q} y(t)\right) d_{q} t \\
& =\frac{-1}{q} \int_{0}^{x} D_{q^{-1}} W_{q}(y, z)(t) d_{q} t \\
& =\lim _{n \rightarrow \infty} W_{q}(y, z)\left(x q^{n}\right)-W_{q}(y, z)\left(x q^{-1}\right) .
\end{aligned}
$$


Since $y, z \in C_{q}^{2}(0, \infty)$, then $y, z, D_{q} y$, and $D_{q} z$ are continuous at zero. Consequently,

$$
\lim _{n \rightarrow \infty} W_{q}(y, z)\left(x q^{n}\right)=W_{q}(y, z)(0),
$$

which completes the proof.

Lemma 2.5. For each $\lambda \in \mathbb{C}$, the $q$-Wronskian $W_{q}(y(\cdot, \lambda), z(\cdot, \lambda))(x)$ is independent of $x, 0 \leq x<\infty$ for any two solutions $y, z$ of equation (1.14). Moreover,

$$
W_{q}(y(\cdot, \lambda), z(\cdot, \lambda))(x) \equiv W_{q}(y(\cdot, \lambda), z(\cdot, \lambda))(0), \quad x \in[0, \infty) .
$$

Proof. Let $\lambda, \lambda^{\prime} \in \mathbb{C}, \lambda \neq \lambda^{\prime}$. Substituting with $L_{t} y=\lambda y$ and $L_{t} z=\lambda^{\prime} z$ in Green's formula (2.9), we conclude that

$$
\begin{aligned}
\left(\lambda^{\prime}-\lambda\right) \int_{0}^{x} y(t, \lambda) z\left(t, \lambda^{\prime}\right) d_{q} t= & W_{q}\left(y(\cdot, \lambda), z\left(\cdot, \lambda^{\prime}\right)\right)(0) \\
& -W_{q}\left(y(\cdot, \lambda), z\left(\cdot, \lambda^{\prime}\right)\right)\left(x q^{-1}\right) .
\end{aligned}
$$

Letting $\lambda^{\prime} \rightarrow \lambda$ and noting that the left-hand side of (2.11) vanishes, we obtain (2.10) as required.

LEMma 2.6. If $y(\cdot, \lambda)$ is a solution of (1.14), then for $\lambda \in \mathbb{C}$, we have for any $b>0$,

$$
\int_{0}^{b}|y(x, \lambda)|^{2} d_{q} x=\frac{1}{2 i v}\left(W_{q}(y, \bar{y})(0, \lambda)-W_{q}(y, \bar{y})\left(b q^{-1}, \lambda\right)\right), \quad v:=\Im(\lambda) .
$$

Proof. The lemma follows directly by substituting with $\lambda^{\prime}=\bar{\lambda}$ and $z(x, \lambda)=y(x, \bar{\lambda})$ in $(2.11)$.

\section{$\S 3$. Classification of singular points}

In this section we introduce the limit-point and limit-circle classifications of the singular point, $x=\infty$ of the $q$-difference equation (1.14). Our analysis will be a $q$-analog of that established in [47] and [52]. We consider families of regular $q$-Sturm-Liouville problems on $\left[0, q^{-n}\right], n \in \mathbb{N}$. Then we define a family of circles that converge either to a point or a circle. For any number $\eta, \phi(\cdot, \lambda)+\eta \theta(\cdot, \lambda)$ is the general solution of $(1.14)$. So for every $n \in \mathbb{N}$, we can choose a number $\eta_{n}$ such that $\phi(\cdot, \lambda)+\eta_{n} \theta(\cdot, \lambda)$ satisfies the boundary condition

$$
y\left(q^{-n}, \lambda\right) \cos \beta+D_{q} y\left(q^{-n}, \lambda\right) \sin \beta=0 .
$$


For $n \in \mathbb{N}$, we denote $\eta_{n}$ by $l_{q^{-n}}(\lambda)$. Then $l_{q^{-n}}(\lambda)$ satisfies the relation

$$
l_{q^{-n}}(\lambda)=-\frac{\phi\left(q^{-n}, \lambda\right) \cos \beta+D_{q} \phi\left(q^{-n}, \lambda\right) \sin \beta}{\theta\left(q^{-n}, \lambda\right) \cos \beta+D_{q} \theta\left(q^{-n}, \lambda\right) \sin \beta} .
$$

Since $\phi(x, \lambda), \theta(x, \lambda), D_{q} \phi(x, \lambda)$, and $D_{q} \theta(x, \lambda)$ are entire functions of $\lambda$, then $l_{q^{-n}}(\lambda)$ is a meromorphic function of $\lambda$. Furthermore, all poles of $l_{q^{-n}}(\lambda)$ are real and simple and lie on the nonnegative part of the real axis of the $\lambda$-plane since these poles are eigenvalues of a regular $q$-Sturm-Liouville problem on $\left[0, q^{-n}\right]$ (see [9], [12]). For $\lambda \in \mathbb{C}$, define

$$
l_{q^{-n}}(\lambda, z)=-\frac{\phi\left(q^{-n}, \lambda\right) z+D_{q} \phi\left(q^{-n}, \lambda\right)}{\theta\left(q^{-n}, \lambda\right) z+D_{q} \theta\left(q^{-n}, \lambda\right)}, \quad z \in \mathbb{C} .
$$

From (2.10), we have

$$
\begin{aligned}
& \phi\left(q^{-n}, \lambda\right) D_{q} \theta\left(q^{-n}, \lambda\right)-\theta\left(q^{-n}, \lambda\right) D_{q} \phi\left(q^{-n}, \lambda\right) \\
& \quad=W_{q}(\phi, \theta)\left(q^{-n}\right)=W_{q}(\phi, \theta)(0) \\
& \quad=1 \neq 0 .
\end{aligned}
$$

From the theory of Möbius transformations (see [22]), (3.3) is a one-to-one conformal mapping in $z$ for every $\lambda$. Therefore, if $\Im(\lambda) \neq 0$, then $l_{q^{-n}}(\lambda, z)$ varies on a circle $C_{q^{-n}}(\lambda)$ with a finite radius in the $l$-plane as $z$ varies over the real axis of the $z$-plane. The following theorem calculates the center and the radius of the circle $C_{q^{-n}}(\lambda)$.

TheOREM 3.1. Let $\lambda \in \mathbb{C}, v:=\Im(\lambda) \neq 0$. Then the center of the circle $C_{q^{-n}}(\lambda), P_{n}(\lambda)$, and its radius, $R_{n}(\lambda)$, are given as follows:

$$
\begin{aligned}
P_{n}(\lambda) & =-\frac{W_{q}\left(\phi\left(q^{-n}, \lambda\right), \bar{\theta}\left(q^{-n}, \lambda\right)\right)}{W_{q}\left(\theta\left(q^{-n}, \lambda\right), \bar{\theta}\left(q^{-n}, \lambda\right)\right)}, \\
R_{n}(\lambda) & =\frac{1}{2|v| \int_{0}^{q^{-n}}|\theta(x, \lambda)|^{2} d_{q} x} .
\end{aligned}
$$

Proof. First, the center of the circle $C_{q^{-n}}(\lambda), P_{n}(\lambda)$, is the symmetric point of the point at $\infty$. Thus, if $z^{\prime}, z^{\prime \prime}$ are in the $z$-plane such that

$$
l_{q^{-n}}\left(\lambda, z^{\prime}\right)=\infty, \quad l_{q^{-n}}\left(\lambda, z^{\prime \prime}\right)=P_{n}(\lambda)
$$


then $z^{\prime}, z^{\prime \prime}$ must be symmetric with respect to the real axis of the $z$-plane; that is, $z^{\prime}=\overline{z^{\prime \prime}}$. But $l_{q^{-n}}\left(\lambda, z^{\prime}\right)=\infty$ if and only if $z^{\prime}=-\left(D_{q} \theta\left(q^{-n}, \lambda\right)\right) /$ $\left(\theta\left(q^{-n}, \lambda\right)\right)$. Therefore, $P_{n}(\lambda)$ is given by

$$
\begin{aligned}
P_{n}(\lambda) & =l_{q^{-n}}\left(\lambda,-\frac{\overline{D_{q} \theta\left(q^{-n}, \lambda\right)}}{\overline{\theta\left(q^{-n}, \lambda\right)}}\right) \\
& =-\frac{\phi\left(q^{-n}, \lambda\right)\left(-\frac{\overline{D_{q} \theta\left(q^{-n}, \lambda\right)}}{\overline{\theta\left(q^{-n}, \lambda\right)}}\right)+D_{q} \phi\left(q^{-n}, \lambda\right)}{\theta\left(q^{-n}, \lambda\right)\left(-\frac{\overline{D_{q} \theta\left(q^{-n}, \lambda\right)}}{\theta\left(q^{-n}, \lambda\right)}\right)+D_{q} \theta\left(q^{-n}, \lambda\right)} \\
& =-\frac{W_{q}\left(\phi\left(q^{-n}, \lambda\right), \bar{\theta}\left(q^{-n}, \lambda\right)\right)}{W_{q}\left(\theta\left(q^{-n}, \lambda\right), \bar{\theta}\left(q^{-n}, \lambda\right)\right)} .
\end{aligned}
$$

Now, the radius of the circle $C_{q^{-n}}(\lambda), R_{n}(\lambda)$, is the distance between the center of $C_{q^{-n}}(\lambda)$ and the point $l_{q^{-n}}(\lambda, 0)$ on $C_{q^{-n}}(\lambda)$. Therefore,

$$
\begin{aligned}
R_{n}(\lambda) & =\left|\frac{D_{q} \phi\left(q^{-n}, \lambda\right)}{D_{q} \theta\left(q^{-n}, \lambda\right)}-\frac{W_{q}\left(\phi\left(q^{-n}, \lambda\right), \bar{\theta}\left(q^{-n}, \lambda\right)\right)}{W_{q}\left(\theta\left(q^{-n}, \lambda\right), \bar{\theta}\left(q^{-n}, \lambda\right)\right)}\right| \\
& =\left|\frac{\bar{\theta}\left(q^{-n}, \lambda\right)}{\theta\left(q^{-n}, \lambda\right)}\right|\left|\frac{W_{q}\left(\phi\left(q^{-n}, \lambda\right), \theta\left(q^{-n}, \lambda\right)\right)}{W_{q}\left(\theta\left(q^{-n}, \lambda\right), \bar{\theta}\left(q^{-n}, \lambda\right)\right)}\right| \\
& =\left|\frac{W_{q}\left(\phi\left(q^{-n}, \lambda\right), \theta\left(q^{-n}, \lambda\right)\right)}{W_{q}\left(\theta\left(q^{-n}, \lambda\right), \bar{\theta}\left(q^{-n}, \lambda\right)\right)}\right|=\frac{1}{\left|W_{q}\left(\theta\left(q^{-n}, \lambda\right), \bar{\theta}\left(q^{-n}, \lambda\right)\right)\right|},
\end{aligned}
$$

since

$$
W_{q}\left(\phi\left(q^{-n}, \lambda\right), \theta\left(q^{-n}, \lambda\right)\right)=W_{q}(\phi(0, \lambda), \theta(0, \lambda))=\sin ^{2} \alpha+\cos ^{2} \alpha=1 .
$$

From Lemma 2.6, we obtain

$$
2 i v \int_{0}^{q^{-n}}|\theta(x, \lambda)|^{2} d_{q} x=-W_{q}\left(\theta\left(q^{-n}, \lambda\right), \bar{\theta}\left(q^{-n}, \lambda\right)\right)
$$

Hence,

$$
\left|W_{q}(\theta, \bar{\theta})\left(q^{-n}, \lambda\right)\right|=2|v| \int_{0}^{1}|\theta(x, \lambda)|^{2} d_{q} x,
$$

completing the proof. 
Next we study the geometric properties of the family of circles $\left\{C_{q^{-n}}(\lambda), n \in\right.$ $\mathbb{N}\}$. We notice that

$$
\int_{0}^{q^{-n}}|\theta(x, \lambda)|^{2} d_{q} x
$$

is a positive number.

THEOREM 3.2. If $v=\Im(\lambda)>0$, then the interior of the circle $C_{q^{-n}}(\lambda)$ is mapped onto the lower half-plane of the z-plane by $l_{q^{-n}}$.

Proof. Since the real axis of the $z$-plane is the image of the circle $C_{q^{-n}}(\lambda)$ by the Möbius transformation (3.3), then the interior of $C_{q^{-n}}(\lambda)$ is mapped either onto the upper half-plane or onto the lower half of the $z$-plane, and further, $\infty$ of the $l$-plane is mapped onto the point $-D_{q} \theta\left(q^{-n}, \lambda\right) / \theta\left(q^{-n}, \lambda\right)$ of the $z$-plane. From the definition of the $q$-Wronskian and equation (3.5), we obtain

$$
\begin{aligned}
\Im\left(-\frac{D_{q} \theta\left(q^{-n}, \lambda\right)}{\theta\left(q^{-n}, \lambda\right)}\right) & =\frac{-i}{2}\left\{-\frac{D_{q} \theta\left(q^{-n}, \lambda\right)}{\theta\left(q^{-n}, \lambda\right)}+\frac{\overline{D_{q} \theta\left(q^{-n}, \lambda\right)}}{\overline{\theta\left(q^{-n}, \lambda\right)}}\right\} \\
& =\frac{-i}{2} \frac{W_{q}(\theta, \bar{\theta})\left(q^{-n}, \lambda\right)}{\left|\theta\left(q^{-n}, \lambda\right)\right|^{2}} \\
& =\frac{v \int_{0}^{q^{-n}}|\theta(x, \lambda)|^{2} d_{q} x}{\left|\theta\left(q^{-n}, \lambda\right)\right|^{2}}>0 .
\end{aligned}
$$

Thus, $-D_{q} \theta\left(q^{-n}, \lambda\right) / \theta\left(q^{-n}, \lambda\right)$ belongs to the upper half-plane of the $z$ plane. Hence, $\infty$, which doesn't belong to the interior of $C_{q^{-n}}(\lambda)$, is mapped into the upper half-plane.

Since $W_{q}(\phi, \theta)\left(q^{-n}, \lambda\right) \neq 0, n \in \mathbb{N}$, then the transformation in (3.3) has a unique inverse, which is

$$
z=-\frac{D_{q} \theta\left(q^{-n}, \lambda\right) l_{q^{-n}}+D_{q} \phi\left(q^{-n}, \lambda\right)}{\theta\left(q^{-n}, \lambda\right) l_{q^{-n}}+\phi\left(q^{-n}, \lambda\right)} .
$$

In view of this, we can prove the following theorem.

THEOREM 3.3. If $v=\Im(\lambda)>0$, then $l$ belongs to the interior of the circle $C_{q^{-n}}(\lambda)$ if and only if

$$
\int_{0}^{q^{-n}}|\phi(x, \lambda)+l \theta(x, \lambda)|^{2} d_{q} x<\frac{\Im(l)}{v},
$$


and l lies on the circle $C_{q^{-n}}(\lambda)$ if and only if

$$
\int_{0}^{q^{-n}}|\phi(x, \lambda)+l \theta(x, \lambda)|^{2} d_{q} x=\frac{\Im(l)}{v} .
$$

Proof. Easy calculations yield, for $l \in \mathbb{C}$,

$$
\begin{aligned}
W_{q}(\phi+l \theta, \overline{\phi+l \theta})(0, \lambda)= & W_{q}(\phi, \bar{\phi})(0, \lambda)+l W_{q}(\theta, \bar{\phi})(0, \lambda) \\
& +\bar{l} W_{q}(\phi, \bar{\theta})(0, \lambda)+|l|^{2} W_{q}(\theta, \bar{\theta})(0, \lambda) \\
= & -l+\bar{l}=-2 i \Im(l) .
\end{aligned}
$$

Applying Lemma 2.6 to the function $\phi+l \theta$ together with the previous equation, we obtain

$$
2 v \int_{0}^{q^{-n}}|\phi(x, \lambda)+l \theta(x, \lambda)|^{2} d_{q} x=2 \Im(l)-i W_{q}(\phi+l \theta, \overline{\phi+l \theta})\left(q^{-n}, \lambda\right) .
$$

By Theorem 3.2, if $v=\Im(\lambda)>0$, then $l$ belongs to the interior of the circle $C_{q^{-n}}(\lambda)$ if $\Im(z)<0$, that is, if $i(z-\bar{z})>0$. From the inverse relation (3.7),

$$
\begin{aligned}
i(z-\bar{z}) & =i\left\{-\frac{D_{q} \theta\left(q^{-n}, \lambda\right) l+D_{q} \phi\left(q^{-n}, \lambda\right)}{\theta\left(q^{-n}, \lambda\right) l+\phi\left(q^{-n}, \lambda\right)}+\frac{D_{q} \bar{\theta}\left(q^{-n}, \lambda\right) \bar{l}+D_{q} \bar{\phi}\left(q^{-n}, \lambda\right)}{\bar{\theta}\left(q^{-n}, \lambda\right) \bar{l}+\bar{\phi}\left(q^{-n}, \lambda\right)}\right\} \\
& =i \frac{W_{q}(\phi+l \theta, \bar{\phi}+l \theta)\left(q^{-n}, \lambda\right)}{\left|\theta\left(q^{-n}, \lambda\right) l+\phi\left(q^{-n}, \lambda\right)\right|^{2}} .
\end{aligned}
$$

Then $\Im(z)<0$ if and only if

$$
i W_{q}(\phi+l \theta, \bar{\phi}+\overline{l \theta})\left(q^{-n}, \lambda\right)>0 .
$$

Comparing (3.11) with (3.10) gives (3.8). On the other hand, $l$ lies on the circle $C_{q^{-n}}(\lambda)$ if and only if $\Im(z)=0$; that is, $i(z-\bar{z})=0$. Hence,

$$
W_{q}(\phi+l \theta, \overline{\phi+l \theta})\left(q^{-n}, \lambda\right)=0 .
$$

Substituting in (3.10) implies (3.9) and the rest of the proof as well.

Theorem 3.3 also holds when $v=\Im(\lambda)<0$. Since $n$ is arbitrary, the previous procedure can be repeated throughout $\mathbb{N}$ to end with a family of circles $\left\{C_{q^{-n}}\right\}_{n \in \mathbb{N}}$ with the following property. 
THEOREM 3.4. If $v=\Im(\lambda) \neq 0$ and $m, n \in \mathbb{N}$ such that $m<n$, then

$$
\overline{C_{q^{-n}}(\lambda)} \subseteq \overline{C_{q^{-m}}(\lambda)},
$$

where $\overline{C_{q^{-j}}(\lambda)}, j \in \mathbb{N}$, is the set composed of the circle $C_{q^{-j}}(\lambda)$ and its interior.

Proof. Let $m, n \in \mathbb{N}$ be such that $m<n$, and let $l$ belong to the interior of $C_{q^{-n}}(\lambda)$. Since

$$
\int_{0}^{q^{-m}}|\phi(x, \lambda)+l \theta(x, \lambda)|^{2} d_{q} x \leq \int_{0}^{q^{-n}}|\phi(x, \lambda)+l \theta(x, \lambda)|^{2} d_{q} x<\frac{\Im(l)}{v},
$$

then $l$ belongs to the interior of $C_{q^{-n}}(\lambda)$, implying that $l$ belongs to the interior of $C_{q^{-m}}(\lambda)$.

REMARK 3.5. Theorem 3.4 and the theorem of nested spheres imply that if $v=\Im(\lambda) \neq 0$, then

$$
C_{\infty}(\lambda):=\lim _{n \rightarrow \infty} C_{q^{-n}}(\lambda)=\bigcap_{n \in \mathbb{N}} \overline{C_{q^{-n}}(\lambda)}
$$

is either a point or a closed circle with a finite radius.

Definition 3.6. The singular point $x=\infty$ is said to be in the limit-point case or in the limit-circle case according to whether $C_{\infty}(\lambda)$ is a point or a circle, respectively.

\section{§4. q-Titchmarsh-Weyl function $m(\lambda)$}

This section involves more investigations on the limit-point, limit-circle classifications and the associated $q$-integrable solutions. We state and prove a condition on $u(\cdot)$ that guarantees that the singularity is of the limit-point type. The $q$-Titchmarsh-Weyl function will be introduced here. We first discuss the independence of the classification with $\lambda$ and $u(\cdot)$. Although according to Definition 3.6 above the classification seems to depend on both $u(\cdot)$ and $\lambda$, we will see that it depends only on $u(\cdot)$, as is shown in the following theorem.

THEOREM 4.1. We have the following.

(i) If, for some $\lambda_{0}, \Im\left(\lambda_{0}\right)=v \neq 0$, every solution of the equation

$$
L_{x} y(x)=\lambda_{0} y(x), \quad 0 \leq x<\infty
$$

is in $\mathcal{H}$, then the point $x=\infty$ is in the limit-circle case for this $\lambda_{0}$. 
(ii) If, for some $\lambda_{0}, \Im\left(\lambda_{0}\right)=v \neq 0$, the point $x=\infty$ is in the limit-circle case, then for every $\lambda$, every solution $y$ of the equation

$$
L_{x} y(x)=\lambda y(x), \quad 0 \leq x<\infty
$$

is in $\mathcal{H}$.

Proof. We start by proving (i). Since $\theta\left(\cdot, \lambda_{0}\right)$ is a nontrivial solution of the equation (4.1), then by assumption,

$$
0<\int_{0}^{\infty}\left|\theta\left(x, \lambda_{0}\right)\right|^{2} d_{q} x<\infty
$$

Hence, the radii $R_{n}$ of the circles $C_{q^{-n}}\left(\lambda_{0}\right)$ approach a positive limit as $n \longrightarrow \infty$; that is, $x=\infty$ is in the limit-circle case for this $\lambda_{0}$. To prove (ii), let $x=\infty$ be in the limit-circle case for some $\lambda_{0}, \Im\left(\lambda_{0}\right)=v \neq 0$. We first prove that every solution $z\left(\cdot, \lambda_{0}\right)$ of $(4.2)$ when $\lambda=\lambda_{0}$ is in $\mathcal{H}$. Indeed, if $l_{1}$ and $l_{2}$ are two distinct points on $C_{\infty}\left(\lambda_{0}\right)$, then the functions $\left\{z_{i}\left(\cdot, \lambda_{0}\right)\right\}_{i=1}^{2}$ defined by

$$
z_{i}\left(x, \lambda_{0}\right):=\phi\left(x, \lambda_{0}\right)+l_{i} \theta\left(x, \lambda_{0}\right), \quad i=1,2
$$

form a fundamental set of solutions of (4.1). Moreover, from Theorem 3.3, we have

$$
\begin{aligned}
\int_{0}^{\infty} & \left|z_{i}\left(x, \lambda_{0}\right)\right|^{2} d_{q} x \\
\quad & =\int_{0}^{\infty}\left|\theta\left(x, \lambda_{0}\right) l_{i}+\phi\left(x, \lambda_{0}\right)\right|^{2} d_{q} x \leq \frac{\Im\left(l_{i}\right)}{v}<\infty, \quad i=1,2 .
\end{aligned}
$$

That is, $z_{i}\left(\cdot, \lambda_{0}\right) \in \mathcal{H}, i=1,2$. Consequently every solution $z\left(\cdot, \lambda_{0}\right)$ of $(4.1)$ is in $\mathcal{H}$. Next we prove that, for any $\lambda$, every solution of $(4.2)$ is in $\mathcal{H}$. To prove this, let $z_{1}(\cdot, \lambda)$ and $z_{2}(\cdot, \lambda)$ be two linearly independent solutions of (4.1) for arbitrary $\lambda$ such that $W_{q}\left(z_{1}(0, \lambda), z_{1}(0, \lambda)\right)=1$. Using the method of variation of parameters (see [5]), the solution of the initial value problem

$$
-\frac{1}{q} D_{q^{-1}} D_{q} z(x)+\{-\lambda+u(x)\} z(x)=f(x), \quad z(0)=D_{q} z(0)=0,
$$

where $f$ is any function in $\mathcal{H}$, is given by

$$
z(x)=c_{1}(x) z_{1}(x, \lambda)+c_{2}(x) z_{2}(x, \lambda),
$$


and $c_{1}(\cdot)$ and $c_{2}(\cdot)$ satisfy the first-order $q$-difference equations

$$
D_{q, x} c_{1}(x)=\frac{q z_{2}(q x, \lambda) f(q x)}{W_{q}\left(z_{1}, z_{2}\right)(x, \lambda)}, \quad D_{q, x} c_{2}(x)=-\frac{q z_{1}(q x, \lambda) f(q x)}{W_{q}\left(z_{1}, z_{2}\right)(x, \lambda)} .
$$

But $W_{q}\left(z_{1}, z_{2}\right)(x, \lambda) \equiv W_{q}\left(z_{1}, z_{2}\right)(0, \lambda)=1$; consequently,

$$
D_{q, x} c_{1}(x)=q z_{2}(q x, \lambda) f(q x), \quad D_{q, x} c_{2}(x)=-q z_{1}(q x, \lambda) f(q x) .
$$

Solving this first-order system, we obtain

$$
\begin{aligned}
& c_{1}(x)=c_{1}(0)+q \int_{0}^{x} z_{2}(q t, \lambda) f(q t) d_{q} t, \\
& c_{2}(x)=c_{2}(0)-q \int_{0}^{x} z_{1}(q t, \lambda) f(q t) d_{q} t .
\end{aligned}
$$

Thus,

$$
\begin{aligned}
z(x)= & \left(c_{1}(0)+q \int_{0}^{x} z_{2}(q t, \lambda) f(q t) d_{q} t\right) z_{1}(x, \lambda) \\
& +\left(c_{2}(0)-q \int_{0}^{x} z_{1}(q t, \lambda) f(q t) d_{q} t\right) z_{2}(x, \lambda) .
\end{aligned}
$$

From the initial conditions $z(0)=D_{q} z(0)=0$, we obtain the equations

$$
\begin{aligned}
& 0=c_{1}(0) z_{1}(0, \lambda)+c_{2}(0) z_{2}(0, \lambda), \\
& 0=c_{1}(0) D_{q} z_{1}(0, \lambda)+c_{2}(0) D_{q} z_{2}(0, \lambda) .
\end{aligned}
$$

Since $W_{q}\left(z_{1}, z_{2}\right)(0) \neq 0$, then $c_{1}(0)=c_{2}(0)=0$. Hence,

$$
z(x)=(K f)(x)=q \int_{0}^{x} k(x, q s, \lambda) f(q s) d_{q} s=\int_{0}^{x} k(x, s, \lambda) f(s) d_{q} s
$$

where $k(x, s, \lambda)$ is the kernel

$$
k(x, s, \lambda)=z_{1}(x, \lambda) z_{2}(s, \lambda)-z_{2}(x, \lambda) z_{1}(s, \lambda) .
$$

Now let $z(x, \lambda)$ be a solution of the initial value problem

$$
L_{x} z=\lambda z, \quad z(0)=\gamma, D_{q} z(0)=\delta, 0 \leq x<\infty .
$$

Since for each fixed $x, z(x, \lambda)$ is an entire function of $\lambda$, then $z(x, \lambda)$ can be expanded in the Taylor series 


$$
z(x, \lambda)=z_{0}(x)+\left(\lambda-\lambda_{0}\right) z_{1}(x)+\left(\lambda-\lambda_{0}\right)^{2} z_{2}(x)+\cdots .
$$

In the following we prove that the functions $z_{i}(x), i=1,2, \ldots$ are solutions of the system of initial value problems

$$
L_{x} z_{n}-\lambda_{0} z_{n}=z_{n-1}, \quad z_{n}(0)=0, D_{q} z_{n}(0)=0, n \geq 1 .
$$

First of all, the initial conditions

$$
z_{0}(0)=\gamma, \quad D_{q} z_{0}(0)=\delta ; \quad z_{n}(0)=D_{q} z_{n}(0)=0, \quad n \geq 1 .
$$

result directly from (4.8). Applying $L_{x}$ to (4.8), we obtain

$$
L_{x} z(x, \lambda)=L_{x} z_{0}(x)+L_{x}\left\{\left(\lambda-\lambda_{0}\right) z_{1}(x)+\left(\lambda-\lambda_{0}\right)^{2} z_{2}(x)+\cdots\right\} .
$$

By equation (4.7), we have

$$
L_{x} z(x, \lambda)=\lambda z(x)=\lambda z_{0}(x)+\lambda\left\{\left(\lambda-\lambda_{0}\right) z_{1}(x)+\left(\lambda-\lambda_{0}\right)^{2} z_{2}(x)+\cdots\right\} .
$$

Subtracting (4.7) from (4.11) and substituting with $\lambda=\lambda_{0}$, we obtain

$$
L_{x} z_{0}=\lambda_{0} z_{0}, \quad z_{0}(0)=\gamma, D_{q} z_{0}(0)=\delta, 0 \leq x<\infty .
$$

Now substituting from (4.12) in (4.10) and using (4.11) yield

$$
\begin{aligned}
0= & \left(\lambda_{0}-\lambda\right) z_{0}(x)+\left(\lambda-\lambda_{0}\right) L_{x} z_{1}(x)-\lambda\left(\lambda-\lambda_{0}\right) z_{1}(x) \\
& +L_{x}\left\{\left(\lambda-\lambda_{0}\right)^{2} z_{2}(x)+\left(\lambda-\lambda_{0}\right)^{3} z_{3}(x)+\cdots\right\} \\
& -\lambda\left\{\left(\lambda-\lambda_{0}\right)^{2} z_{2}(x)+\left(\lambda-\lambda_{0}\right)^{3} z_{3}(x)+\cdots\right\} .
\end{aligned}
$$

Dividing on $\left(\lambda-\lambda_{0}\right), \lambda \neq \lambda_{0}$, and substituting again with $\lambda=\lambda_{0}$ give

$$
L_{x} z-\lambda_{0} z=z_{0} .
$$

Continuing this procedure, we obtain the system of initial value problems (4.9). From (4.6) we have

$$
z_{n}(x)=\left(K z_{n-1}\right)(x), \quad n=1,2, \ldots
$$

Since

$$
\int_{0}^{\infty}\left|z_{i}\left(x, \lambda_{0}\right)\right|^{2} d_{q} x<\infty, \quad i=1,2
$$


then

$$
\int_{0}^{\infty} k\left(x, \lambda_{0}\right) d_{q} x<\infty, \quad k\left(x, \lambda_{0}\right)=\int_{0}^{x}\left|K\left(x, s, \lambda_{0}\right)\right|^{2} d_{q} s .
$$

We prove by induction on $m$ that

$$
\begin{aligned}
\left|z_{n}\left(q^{m}\right)\right|^{2} \leq & \frac{\left\|z_{0}\right\|^{2}}{n !} k\left(q^{m+1}, \lambda_{0}\right) \\
& \times \int_{0}^{q^{m+1}} \int_{0}^{x_{1}} \int_{0}^{x_{2}} \cdots \int_{0}^{x_{n-2}} k\left(x_{1}, \lambda_{0}\right) \\
& \times k\left(x_{2}, \lambda_{0}\right) \cdots k\left(x_{n}, \lambda_{0}\right) d x_{1} d x_{2} \cdots d x_{n-1},
\end{aligned}
$$

$n \geq 2, m \in \mathbb{Z}$, where hereafter $\|\cdot\|$ denotes the norm of $\mathcal{H}$. By CauchySchwarz's inequality, we obtain for $m \in \mathbb{Z}$,

$$
\begin{aligned}
\left|z_{1}\left(q^{m}\right)\right|^{2} & =\int_{0}^{q^{m+1}} k\left(q^{m}, s, \lambda_{0}\right) z_{0}(s) d_{q} s \\
& \leq\left\|z_{0}\right\|^{2} \int_{0}^{q^{m+1}}\left|k\left(q^{m}, s, \lambda_{0}\right)\right|^{2} d_{q} s \leq\left\|z_{0}\right\|^{2} k\left(q^{m}, \lambda_{0}\right) .
\end{aligned}
$$

Also,

$$
\begin{aligned}
\left|z_{2}\left(q^{m}\right)\right|^{2} & =\left|\int_{0}^{q^{m+1}} k\left(q^{m}, s, \lambda_{0}\right) z_{1}(s) d_{q} s\right|^{2} \\
& \leq\left\|z_{0}\right\|^{2}\left(\int_{0}^{q^{m+1}}\left|k\left(q^{m}, s, \lambda_{0}\right)\right|^{2} d_{q} s\right)\left(\int_{0}^{q^{m+1}} k\left(s, \lambda_{0}\right) d_{q} s\right) \\
& \leq\left\|z_{0}\right\|^{2} k\left(q^{m}, \lambda_{0}\right) \int_{0}^{q^{m}} k\left(s, \lambda_{0}\right) d s .
\end{aligned}
$$

Suppose that (4.16) holds for $n \in \mathbb{N}$. Then using (4.14) and the induction hypothesis, we obtain

$$
\begin{aligned}
\left|z_{n+1}\left(q^{m}\right)\right|^{2} & =\left|\int_{0}^{q^{m+1}} k\left(x, s, \lambda_{0}\right) z_{n}(s) d_{q} s\right|^{2} \\
& \leq\left(\int_{0}^{q^{m+1}}\left|k\left(x, s, \lambda_{0}\right)\right|^{2} d_{q} s\right)\left(\int_{0}^{q^{m+1}}\left|z_{n}(s)\right|^{2} d_{q} s\right) \\
& \leq k\left(q^{m}, \lambda_{0}\right) \int_{0}^{q^{m+1}}\left|z_{n}(s)\right|^{2} d_{q} s
\end{aligned}
$$




$$
\begin{aligned}
\leq & \frac{\left\|z_{0}\right\|^{2} k\left(q^{m}, \lambda_{0}\right)}{n !} \int_{0}^{q^{m+1}} \int_{0}^{x_{1}} \int_{0}^{x_{2}} \cdots \int_{0}^{x_{n-1}} k\left(x_{1}, \lambda_{0}\right) \\
& \times k\left(x_{2}, \lambda_{0}\right) \cdots k\left(x_{n}, \lambda_{0}\right) d_{q} t d_{q} x_{1} \cdots d_{q} x_{n} .
\end{aligned}
$$

Hence, (4.16) holds for all $n \in \mathbb{N}, n \geq 2$, and $m \in \mathbb{Z}$. Set

$$
\varepsilon:=\int_{0}^{\infty} k\left(s, \lambda_{0}\right) d_{q} s
$$

then

$$
\begin{aligned}
& \left|\int_{0}^{q^{m+1}} \quad \int_{0}^{x_{1}} \int_{0}^{x_{2}} \cdots \int_{0}^{x_{n-2}} k\left(x_{1}, \lambda_{0}\right) k\left(x_{2}, \lambda_{0}\right) \cdots k\left(x_{n}, \lambda_{0}\right) d x_{1} d x_{2} \cdots d x_{n-1}\right| \\
& \leq\left(\int_{0}^{\infty} k\left(s, \lambda_{0}\right) d_{q} s\right)^{n-1}=\epsilon^{n-1}, \quad n=2,3, \ldots
\end{aligned}
$$

One can also verify that the previous inequality holds also at $n=1$. Thus,

$$
\sum_{m=-\infty}^{\infty} q^{m}(1-q)\left|z_{n}\left(q^{m}\right)\right|^{2} \leq \frac{\varepsilon^{n-1}}{n !} \sum_{m=-\infty}^{\infty} q^{m}(1-q) k\left(q^{m}, \lambda_{0}\right)=\frac{\varepsilon^{n}}{n !} .
$$

That is, $z_{n} \in \mathcal{H}$ for all $n \in \mathbb{N}$. From (4.8), we obtain

$$
\|z\| \leq\left\|z_{0}\right\|+\left|\lambda-\lambda_{0}\right|\left\|z_{1}\right\|+\left|\lambda-\lambda_{0}\right|^{2}\left\|z_{2}\right\|+\cdots \leq\left\|z_{0}\right\| \sum_{n=0}^{\infty}\left|\lambda-\lambda_{0}\right|^{n} \frac{\varepsilon^{n}}{n !} .
$$

The previous series is absolutely convergent for all $\lambda \in \mathbb{C}$. Hence, $z(\cdot, \lambda) \in \mathcal{H}$.

In the following we define the Titchmarsh-Weyl function $m(\lambda)$ associated with (1.14). As in the classical case, it will be a single-valued function in the limit-point case, and it is a multivalued function in the limit-circle case.

THEOREM 4.2. Let $m=m(\lambda)$ be the limit-point or any point on the limit circle. Then, for every nonreal $\lambda$, the solution

$$
\psi(x, \lambda):=\phi(x, \lambda)+m(\lambda) \theta(x, \lambda)
$$

of (1.14) belongs to $\mathcal{H}$.

Proof. From Theorem 3.3 above, we have for every $n \in \mathbb{N}$,

$$
\int_{0}^{q^{-n}}|\phi(x, \lambda)+m(\lambda) \theta(x, \lambda)|^{2} d_{q} x<\frac{\Im(m(\lambda))}{v} .
$$


Then letting $n \longrightarrow \infty$, we obtain

$$
\int_{0}^{\infty}|\phi(x, \lambda)+m(\lambda) \theta(x, \lambda)|^{2} d_{q} x \leq \frac{\Im(m(\lambda))}{v} .
$$

Thus,

$$
\psi(x, \lambda)=\phi(x, \lambda)+m(\lambda) \theta(x, \lambda) \in \mathcal{H} .
$$

Notice that in the limit-circle case, since $R_{n}(\lambda)$ tends to positive values as $n \longrightarrow \infty$, then by $(3.4), \theta(x, \lambda) \in \mathcal{H}$. Consequently,

$$
\phi(x, \lambda)=\psi(x, \lambda)-m(\lambda) \theta(x, \lambda) \in \mathcal{H} .
$$

Hence, every solution of (1.14) belongs to $\mathcal{H}$. The function $m(\lambda)$ is called the Titchmarsh-Weyl function. In the classical Sturm-Liouville theory, there are many studies concerning possible necessary and/or sufficient conditions on $u(\cdot)$ to classify the type of the singular points. A useful sufficient condition for the operator $L_{x}$ to be in the limit-point case is given in the following theorem. We first prove the following lemma.

LEMMA 4.3. Let $u\left(q^{m}\right) \geq-k\left(1+q^{2 m}\right)$ for all $m \in \mathbb{Z}$, where $k$ is a positive number. If $\phi \in \mathcal{H}$ is a solution of the q-difference equation $L_{x} y=0$, then $\left(D_{q} \phi(x)\right) /(1+q x) \in \mathcal{H}$.

Proof. Let $\phi \in \mathcal{H}$ be a solution of the $q$-difference equation $L_{x} y=0$; then

$$
-\frac{1}{q} D_{q^{-1}} D_{q} \phi(x)+u(x) \phi(x)=0, \quad x \in(0, \infty) .
$$

Since $D_{q^{-1}} D_{q} \phi(x)=D_{q}^{2} \phi\left(q^{-1} x\right)$, then replacing $x$ by $q x$ in the last equality gives

$$
-D_{q}^{2} \phi(x)+q u(q x) \phi(q x)=0, \quad x \in(0, \infty)
$$

Hence,

$$
\begin{aligned}
\int_{0}^{q^{-n}} \frac{D_{q}^{2} \phi(x) \phi(q x)}{(1+q x)^{2}} d_{q} x & =q \int_{0}^{q^{-n}} \frac{u(q x) \phi^{2}(q x)}{(1+q x)^{2}} d_{q} x \\
& \geq-q k \int_{0}^{q^{-n}} \phi^{2}(q x) d_{q} x \\
& >-q k \int_{0}^{\infty} \phi^{2}(q x) d_{q} x=k_{1} .
\end{aligned}
$$


Applying $q$-integration by parts to

$$
\int_{0}^{q^{-n}} \frac{D_{q}^{2} \phi(x) \phi(q x)}{(1+q x)^{2}} d_{q} x
$$

we obtain

$$
\begin{aligned}
-\int_{0}^{q^{-n}} \frac{D_{q}^{2} \phi(x) \phi(q x)}{(1+q x)^{2}} d_{q} x= & \phi(0) D_{q} \phi(0)-\frac{\phi\left(q^{-n}\right) D_{q} \phi\left(q^{-n}\right)}{\left(1+\left(q^{-n}\right)\right)^{2}} \\
& +\int_{0}^{q^{-n}} D_{q} \phi(x) D_{q}\left(\frac{\phi(x)}{(1+x)^{2}}\right) d_{q} x .
\end{aligned}
$$

From (1.9), we obtain

$$
\begin{aligned}
-\int_{0}^{q^{-n}} \frac{D_{q}^{2} \phi(x) \phi(q x)}{(1+q x)^{2}} d_{q} x= & -\frac{\phi\left(q^{-n}\right) D_{q} \phi\left(q^{-n}\right)}{\left(1+\left(q^{-n}\right)\right)^{2}} \\
& +\int_{0}^{q^{-n}} \frac{\left(D_{q} \phi(x)\right)^{2}}{(1+q x)^{2}} d_{q} x+\phi(0) D_{q} \phi(0) \\
& -\int_{0}^{q^{-n}} \frac{\phi(x) D_{q} \phi(x)(2+x(1+q))}{(1+x)^{2}(1+q x)^{2}} d_{q} x
\end{aligned}
$$

Set

$$
k_{2}:=k_{1}-\phi(0) D_{q} \phi(0), \quad H_{n}:=\int_{0}^{q^{-n}} \frac{\left(D_{q} \phi(x)\right)^{2}}{(1+q x)^{2}} d_{q} x .
$$

Inequality (4.19) leads to

$$
-\frac{\phi\left(q^{-n}\right) D_{q} \phi\left(q^{-n}\right)}{\left(1+\left(q^{-n}\right)\right)^{2}}+H_{n}-\int_{0}^{q^{-n}} \frac{\phi(x) D_{q} \phi(x)(2+x(1+q))}{(1+x)^{2}(1+x)^{2}} d_{q} x<k_{2} .
$$

Applying Cauchy-Schwarz's inequality to the $q$-integral on the left-hand side of (4.21) yields

$$
\left|\int_{0}^{q^{-n}} \frac{\phi(x) D_{q} \phi(x)(2+x(1+q))}{(1+x)^{2}(1+q x)^{2}} d_{q} x\right|^{2}
$$

$$
\leq\left(\int_{0}^{q^{-n}}|\phi(x)|^{2} d_{q} x\right)\left(\int_{0}^{q^{-n}}\left|D_{q} \phi(x)\right|^{2}\left(\frac{(2+x(1+q))}{(1+x)^{2}(1+q x)^{2}}\right)^{2} d_{q} x\right) .
$$


Since

$$
\frac{(2+x(1+q))}{(1+x)^{2}(1+q x)^{2}} \leq \frac{2}{1+q x}, \quad x>0,
$$

then

$$
\begin{aligned}
\int_{0}^{q^{-n}} & \left|D_{q} \phi(x)\right|^{2}\left(\frac{(2+x(1+q))}{(1+x)^{2}(1+q x)^{2}}\right)^{2} d_{q} x \\
& \leq \int_{0}^{q^{-n}}\left|D_{q} \phi(x)\right|^{2}\left(\frac{2}{(1+q x)}\right)^{2} d_{q} x \\
& =4 H_{n} .
\end{aligned}
$$

Substituting from the last inequality in (4.22) implies that

$$
\begin{aligned}
\mid \int_{0}^{q^{-n}} & \left.\frac{\phi(x) D_{q} \phi(x)(2+x(1+q))}{(1+x)^{2}(1+q x)^{2}} d_{q} x\right|^{2} \\
& \leq 4 H_{n} \int_{0}^{q^{-n}}|\phi(x)|^{2} d_{q} x \\
& \leq 4 H_{n} \int_{0}^{\infty}|\phi(x)|^{2} d_{q} x
\end{aligned}
$$

that is, that

$$
-k_{3} H_{n}^{1 / 2}<\int_{0}^{q^{-n}} \frac{\phi(x) D_{q} \phi(x)(2+x(1+q))}{(1+x)^{2}(1+q x)^{2}} d_{q} x<k_{3} H_{n}^{1 / 2},
$$

where $k_{3}=2\|\phi\|$. Combining the last inequality and (4.21), we obtain

$$
-\frac{\phi\left(b q^{-n}\right) D_{q} \phi\left(q^{-n}\right)}{\left(1+\left(q^{-n}\right)\right)^{2}}+H_{n}-k_{3} H_{n}^{1 / 2}<k_{2} .
$$

Suppose, on the contrary, that $\left(D_{q} \phi(x)\right) /(1+q x) \notin \mathcal{H}$, that is, that $H_{n} \rightarrow \infty$ as $n \rightarrow \infty$. Then

$$
\lim _{n \rightarrow \infty} \frac{\left(H_{n}-k_{3} H_{n}^{1 / 2}-k_{2}\right)}{H_{n}}=1 .
$$

It follows that

$$
\frac{\phi\left(q^{-n}\right) D_{q} \phi\left(q^{-n}\right)}{\left(1+\left(q^{-n}\right)\right)^{2}}>\frac{1}{2} H_{n}
$$


for sufficiently large $n$. Substituting with

$$
D_{q} \phi\left(q^{-n}\right)=\frac{1-\frac{\phi\left(q^{-n+1}\right)}{\phi\left(q^{-n}\right)}}{q^{-n}(1-q)} \phi\left(q^{-n}\right)
$$

in (4.23) gives

$$
\frac{1-\frac{\phi\left(q^{-n+1}\right)}{\phi\left(q^{-n}\right)}}{q^{-n}(1-q)\left(1+\left(q^{-n}\right)\right)^{2}} \phi^{2}\left(q^{-n}\right)>\frac{1}{2} H_{n}
$$

From (4.23), the functions $\phi\left(q^{-n}\right)$ and $D_{q} \phi\left(q^{-n}\right)$ have the same sign for large $n$. Consequently, $0<1-\left(\phi\left(q^{-n+1}\right)\right) /\left(\phi\left(q^{-n}\right)\right)<1$ for sufficiently large $n$. Therefore,

$$
q^{n} \phi^{2}\left(q^{-n}\right)>\frac{(1-q)}{2} H_{n}
$$

for sufficiently large $n$. That is,

$$
\lim _{n \rightarrow \infty} q^{n} \phi^{2}\left(q^{-n}\right)=\infty
$$

This contradicts the assumption that $\phi \in \mathcal{H}$.

THEOREM 4.4. If $u\left(q^{m}\right) \geq-k\left(1+q^{m}\right)^{2}$, where $k$ is a positive constant and $m \in \mathbb{Z}$, then the operator $L_{x}$ is in the limit-point case.

Proof. We prove the theorem by showing that the equation $L_{x} y=0$ does not have two linearly independent solutions in $\mathcal{H}$. Suppose the contrary, and let $\phi(\cdot), \psi(\cdot) \in \mathcal{H}$ be two real linearly independent solutions of the equation $L_{x} y=0$ in $\mathcal{H}$. We also can assume without any loss of generality that $W_{q}(\phi, \psi)(x) \equiv 1$ for all $x \in[0, \infty)$. Consequently,

$$
\phi(x) D_{q} \psi(x)-\psi(x) D_{q} \phi(x) \equiv 1
$$

dividing by $(1+q x)$ yields

$$
\phi(x) \frac{D_{q} \psi(x)}{(1+q x)}-\psi(x) \frac{D_{q} \phi(x)}{(1+q x)}=\frac{1}{(1+q x)} .
$$

Then,

$$
\int_{0}^{\infty} \frac{1}{(1+q x)} d_{q} x=\int_{0}^{\infty} \phi(x) \frac{D_{q} \psi(x)}{(1+q x)} d_{q} x-\int_{0}^{\infty} \psi(x) \frac{D_{q} \phi(x)}{(1+q x)} d_{q} x .
$$


Applying the triangle and Cauchy-Schwarz inequalities yields

$$
\begin{aligned}
\left|\int_{0}^{\infty} \frac{1}{(1+q x)} d_{q} x\right| \leq & \left|\int_{0}^{\infty} \phi(x) \frac{D_{q} \psi(x)}{(1+q x)} d_{q} x\right|+\left|\int_{0}^{\infty} \psi(x) \frac{D_{q} \phi(x)}{(1+q x)} d_{q} x\right| \\
\leq & \left(\int_{0}^{\infty}|\phi(x)|^{2} d_{q} x\right)^{\frac{1}{2}}\left(\int_{0}^{\infty} \frac{\left|D_{q} \psi(x)\right|^{2}}{(1+q x)^{2}} d_{q} x\right)^{\frac{1}{2}} \\
& +\left(\int_{0}^{\infty}|\psi(x)|^{2} d_{q} x\right)^{\frac{1}{2}}\left(\int_{0}^{\infty} \frac{\left|D_{q} \phi(x)\right|^{2}}{(1+q x)^{2}} d_{q} x\right)^{\frac{1}{2}} .
\end{aligned}
$$

From Lemma 4.3, the functions $\left|D_{q} \phi(x)\right| /(1+q x)$ and $\left|D_{q} \psi(x)\right| /(1+q x)$ are $\mathcal{H}$ functions. Then so is $1 /(1+q x)$, which is a contradiction, and the theorem follows.

LEMMA 4.5. The function $m(\lambda)$ is an analytic in the upper (or lower) half of the $\lambda$-plane. Moreover,

$$
m(\lambda)=O\left(\frac{1}{v}\right), \quad v=\Im(\lambda), \quad \text { as } v \longrightarrow 0 .
$$

Consequently, if $m(\lambda)$ has real poles, they must be simple.

Proof. Since the poles of $l_{q^{-n}}(\lambda)$ are the zeros of $\theta\left(q^{-n}, \lambda\right) \cos \beta+D_{q} \times$ $\theta\left(q^{-n}, \lambda\right) \sin \beta$, then for a given $\beta, l_{q^{-n}}(\lambda)$ is a meromorphic function whose poles lie on the real axis. Also, on the circle $C_{q^{-n}}($ if $v>0$ ),

$$
\int_{0}^{q^{-n}}\left|\phi(x, \lambda)+l_{q^{-n}}(\lambda) \theta(x, \lambda)\right|^{2} d_{q} x=-\frac{\Im\left(l_{q^{-n}}\right)}{v} \leq \frac{\left|l_{q^{-n}}(\lambda)\right|}{v} .
$$

On the other hand, from (1.11), we have

$$
\begin{aligned}
& \int_{0}^{q^{-n}} \quad\left|\phi(x, \lambda)+l_{q^{-n}}(\lambda) \theta(x, \lambda)\right|^{2} d_{q} x \\
& \quad \geq \frac{1}{2}\left|l_{q^{-n}}\right|^{2} \int_{0}^{q^{-n}}\left|\theta^{2}(x)\right| d_{q} x-\int_{0}^{q^{-n}}\left|\phi^{2}(x)\right| d_{q} x .
\end{aligned}
$$

Combining this inequality with (4.28), we obtain

$$
\frac{\left|l_{q^{-n}}\right|}{v} \geq \frac{1}{2}\left|l_{q^{-n}}\right|^{2} \int_{0}^{q^{-n}}\left|\theta^{2}(t)\right| d_{q} t-\int_{0}^{q^{-n}}\left|\phi^{2}(t)\right| d_{q} t
$$


for every $n \in \mathbb{N}$. Solving the last quadratic inequality for $\left|l_{q^{-n}}(\lambda)\right|$ gives

$$
\begin{aligned}
\left|l_{q^{-n}}(\lambda)\right| \leq & \frac{1}{v \int_{0}^{q^{-n}}|\theta(x, \lambda)|^{2} d_{q} x} \\
& +\left(\frac{2 \int_{0}^{q^{-n}}|\phi(x, \lambda)|^{2} d_{q} x}{\int_{0}^{q^{-n}}|\theta(x, \lambda)|^{2} d_{q} x}+\frac{1}{v^{2}\left(\int_{0}^{q^{-n}}|\theta(x, \lambda)|^{2} d_{q} x\right)^{2}}\right)^{\frac{1}{2}}
\end{aligned}
$$

From the theory developed in [9], the poles of every $l_{q^{-n}}$ are real and simple. For a given nonreal $\lambda$, the plane is covered with circles $C_{q^{-n}}$ under the effect of $l_{q^{-n}}$, and these circles shrink as $n$ increases. Therefore, the family $\left\{l_{q^{-n}}\right\}_{n \in \mathbb{N}}$ is uniformly bounded on each bounded domain in the $\lambda$-plane which lies entirely in the upper (or lower) half $\lambda$-plane; that is, $\left\{l_{q^{-n}}\right\}_{n \in \mathbb{N}}$ is a normal family (see, e.g., [6]). Hence, $\left\{l_{q^{-n}}\right\}_{n \in \mathbb{N}}$ approaches an analytic function $m(\lambda)$ in the upper (or lower) half $\lambda$-plane in the limit-point case. If (1.14) is in the limit-circle case, we pick a subsequence $\left\{q^{-n_{k}}\right\}_{k \in \mathbb{N}}$ and independent numbers $\beta_{k}$ such that $\left\{l_{q^{-n_{k}}}\right\}_{n \in \mathbb{N}}$ with this $\beta_{k}$ has a limit $m(\lambda)$. For the same reasons, $m(\lambda)$ is analytic in the upper (or lower) half $\lambda$-plane. The right-hand side of (4.29) has the asymptotic relation $O(1 / v)$ as $v \rightarrow 0$; it follows that $m(\lambda)=O(1 / v)$. If $\lambda_{n}$ is a real pole of $m(\lambda)$, then for sufficiently large $v, \lambda=\lambda_{n}+i v, v=-i\left(\lambda-\lambda_{n}\right)$, and

$$
\left(\lambda-\lambda_{n}\right) m(\lambda)=O(1), \quad \text { as } v \rightarrow \infty .
$$

Therefore, $\lambda_{n}$ must be simple.

The functions denoted by $m(\lambda)$ in the upper and lower half-planes are not necessarily analytic continuations of each other. In this paper we assume that $m(\lambda)$ is a single meromorphic function whose singularities are poles on the real axis. Let us denote these poles by $\lambda_{0}, \lambda_{1}, \lambda_{2}, \ldots$, and their corresponding residues by $r_{0}, r_{1}, r_{2}, \ldots$; that is,

$$
r_{k}:=\lim _{\lambda \rightarrow \lambda_{k}}\left(\lambda-\lambda_{k}\right) m(\lambda), \quad k \in \mathbb{N}_{0}
$$

The set $\left\{r_{n}\right\}_{r=0}^{\infty}$ is a set of real numbers. Indeed, using (3.2) and Lemma 2.3,

$$
\overline{r_{k}}=\overline{\lim _{\lambda \rightarrow \lambda_{k}}\left(\lambda-\lambda_{k}\right) m(\lambda)}=\lim _{\bar{\lambda} \rightarrow \lambda_{k}}\left(\bar{\lambda}-\lambda_{k}\right) m(\bar{\lambda})=r_{n}, \quad n=0,1, \ldots
$$




\section{$\S 5 . \quad$ Eigenfunction expansions}

In this section we prove eigenfunction expansion theorems in $\mathcal{H}$. We start with some preliminary results.

Lemma 5.1. Let $\lambda$ and $\lambda^{\prime}$ be fixed nonreal numbers. Then

$$
\lim _{n \rightarrow \infty} W_{q}\left(\psi(\cdot, \lambda), \psi\left(\cdot, \lambda^{\prime}\right)\right)\left(q^{-n}\right)=0 .
$$

Proof. Let $n \in \mathbb{N}$. Since the function $\phi(\cdot, \lambda)+l_{q^{-n}}(\lambda) \theta(\cdot, \lambda)$ satisfies the boundary condition (3.1) at $x=q^{-n}$, independent of $\lambda$, then

$$
W_{q}\left(\phi(\cdot, \lambda)+l_{q^{-n}}(\lambda) \theta(\cdot, \lambda), \phi\left(\cdot, \lambda^{\prime}\right)+l_{q^{-n}}\left(\lambda^{\prime}\right) \theta\left(\cdot, \lambda^{\prime}\right)\right)\left(q^{-n}\right)=0 .
$$

From (5.27),

$$
\phi\left(q^{-n}, \eta\right)=\psi\left(q^{-n}, \eta\right)-m(\lambda) \theta\left(q^{-n}, \eta\right), \quad \eta \in\left\{\lambda, \lambda^{\prime}\right\} .
$$

Substituting in (5.2) yields

$W_{q}\left(\left(\psi+\left(l_{q^{-n}}(\lambda)-m(\lambda)\right) \theta\right)(\cdot, \lambda),\left(\psi+\left(l_{q^{-n}}\left(\lambda^{\prime}\right)-m\left(\lambda^{\prime}\right)\right) \theta\right)\left(\cdot, \lambda^{\prime}\right)\right)\left(q^{-n}\right)=0$

That is,

$$
\begin{aligned}
& W_{q}\left(\psi(\cdot, \lambda), \psi\left(\cdot, \lambda^{\prime}\right)\right)\left(q^{-n}\right)+\left\{l_{q^{-n}}(\lambda)-m(\lambda)\right\} W_{q}\left(\theta(\cdot, \lambda), \psi\left(\cdot, \lambda^{\prime}\right)\right)\left(q^{-n}\right) \\
& +\left\{l_{q^{-n}}\left(\lambda^{\prime}\right)-m\left(\lambda^{\prime}\right)\right\} W_{q}\left(\psi(\cdot, \lambda), \theta\left(\cdot, \lambda^{\prime}\right)\right)\left(q^{-n}\right) \\
& +\left\{l_{q^{-n}}(\lambda)-m(\lambda)\right\}\left\{l_{q^{-n}}\left(\lambda^{\prime}\right)-m\left(\lambda^{\prime}\right)\right\} W_{q}\left(\theta(\cdot, \lambda), \theta\left(\cdot, \lambda^{\prime}\right)\right)\left(q^{-n}\right)=0 .
\end{aligned}
$$

Using Green's identity, (2.11),

$$
\begin{aligned}
W_{q}\left(\theta(\cdot, \lambda), \psi\left(\cdot, \lambda^{\prime}\right)\right)\left(q^{-n}\right)= & W_{q}\left(\theta(\cdot, \lambda), \psi\left(\cdot, \lambda^{\prime}\right)\right)(0) \\
& +\left(\lambda^{\prime}-\lambda\right) \int_{0}^{b q^{-n+1}} \theta(x, \lambda) \psi\left(x, \lambda^{\prime}\right) d_{q} x \\
= & O(1)+O\left(\int_{0}^{\infty}|\theta(x, \lambda)|^{2} d_{q} x\right)^{\frac{1}{2}}
\end{aligned}
$$

as $n \rightarrow \infty, \lambda$ and $\lambda^{\prime}$ being fixed. In the limit-point case, we have

$$
\left|l_{q^{-n}}(\lambda)-m(\lambda)\right| \leq 2 R_{n}(\lambda)=\left(v \int_{0}^{q^{-n}}|\theta(x, \lambda)|^{2} d_{q} x\right)^{-1}, \quad \text { for all } n \in \mathbb{N},
$$


so that

$$
\lim _{n \rightarrow \infty}\left|l_{q^{-n}}(\lambda)-m(\lambda)\right| W_{q}\left(\theta(\cdot, \lambda), \psi\left(\cdot, \lambda^{\prime}\right)\right)\left(q^{-n}\right)=0 .
$$

Equation (5.4) also holds in the limit-circle case, if $l_{q^{-n}}(\lambda) \rightarrow m(\lambda)$, since $\theta(\cdot, \lambda)$ is an $\mathcal{H}$-function. Similarly, the other terms in (5.3) tend to zero as $n$ tends to $\infty$, and (5.1) follows.

COROLlary 5.2. If $\lambda$ and $\lambda^{\prime}$ are nonreal numbers, then

$$
\begin{aligned}
\int_{0}^{\infty} \psi\left(x, \lambda^{\prime}\right) \psi(x, \lambda) d_{q} x & =\frac{m\left(\lambda^{\prime}\right)-m(\lambda)}{\lambda-\lambda^{\prime}}, \\
\int_{0}^{\infty}|\psi(x, \lambda)|^{2} d_{q} x & =-\frac{\Im(m(\lambda))}{v} .
\end{aligned}
$$

Proof. From Green's identity, (2.11), we obtain

$$
\begin{aligned}
& \left(\lambda-\lambda^{\prime}\right) \int_{0}^{q^{-n+1}} \psi(x, \lambda) \psi\left(x, \lambda^{\prime}\right) d_{q} x \\
& \quad=W_{q}\left(\psi(\cdot, \lambda), \psi\left(\cdot, \lambda^{\prime}\right)\right)(0)-W_{q}\left(\psi(\cdot, \lambda), \psi\left(\cdot, \lambda^{\prime}\right)\right)\left(q^{-n}\right) .
\end{aligned}
$$

From the initial conditions (2.6) and (2.7), we have

$$
\begin{aligned}
W_{q}\left(\psi(\cdot, \lambda), \psi\left(\cdot, \lambda^{\prime}\right)\right)(0) & =\psi(0, \lambda) D_{q} \psi\left(0, \lambda^{\prime}\right)-\psi\left(0, \lambda^{\prime}\right) D_{q} \psi(0, \lambda) \\
& =m\left(\lambda^{\prime}\right)-m(\lambda)
\end{aligned}
$$

If $\lambda$ and $\lambda^{\prime}$ are not real, then from Lemma 5.1 we have

$$
\lim _{n \rightarrow \infty} W_{q}\left(\psi(\cdot, \lambda), \psi\left(\cdot, \lambda^{\prime}\right)\right)\left(q^{-n}\right)=0
$$

Consequently, taking the limit as $n \rightarrow \infty$ in (5.7) yields (5.5). In particular, taking $\lambda^{\prime}=\bar{\lambda}$ and using Lemma 2.3, we obtain (5.6), and the proof is complete.

Definition 5.3. Let $f$ and $f_{n}, n \in \mathbb{N}$ be functions defined on $\mathbb{R}$. We say that $\left\{f_{n}\right\}_{n=1}^{\infty}$ converges uniformly in $q$-mean to the function $f$ if, for any $X>0$,

$$
\lim _{n \rightarrow \infty} \int_{0}^{X}\left|f_{n}(t)-f(t)\right|^{2} d_{q} t=0 .
$$


LEMMA 5.4. Let $\left\{f_{n}\right\}_{n=1}^{\infty}$ be a sequence of functions in $\mathcal{H}$ which converges uniformly in q-mean to $f$. If there exists a positive real number $K$ such that

$$
\int_{0}^{\infty}\left|f_{n}(x)\right|^{2} d_{q} x \leq K
$$

then $f \in \mathcal{H}$. Moreover, for any function $g \in \mathcal{H}$, we have

$$
\lim _{n \rightarrow \infty} \int_{0}^{\infty} f_{n}(x) g(x) d_{q} x=\int_{0}^{\infty} f(x) g(x) d_{q} x .
$$

Proof. Since $\left\{f_{n}\right\}_{n=1}^{\infty}$ converges to $f$ uniformly in $q$-mean, then given $\varepsilon>0$ and $m \in \mathbb{N}$, there exists $n_{0} \in \mathbb{N}, n_{0}=n_{0}(\varepsilon, m)$ such that

$$
\int_{0}^{q^{-m}}\left|f_{n}(t)-f(t)\right|^{2} d_{q} t<\varepsilon
$$

for all $n \geq n_{0}$. Consequently, from Cauchy-Schwarz's inequality we have

$$
\begin{aligned}
\left(\int_{0}^{q^{-m}}|f(x)|^{2} d_{q} x\right)^{1 / 2} \leq & \left(\int_{0}^{q^{-m}}\left|f_{n_{0}}(x)\right|^{2} d_{q} x\right)^{1 / 2} \\
& +\left(\int_{0}^{q^{-m}}\left|f(x)-f_{n_{0}}(x)\right|^{2} d_{q} x\right)^{1 / 2} \\
\leq & \sqrt{K}+\sqrt{\varepsilon}
\end{aligned}
$$

for all $m \in \mathbb{N}$. Since $\varepsilon$ is arbitrary, then taking the limit as $m \rightarrow \infty$ yields

$$
\int_{0}^{\infty}|f(x)|^{2} d_{q} x \leq K
$$

hence, $f \in \mathcal{H}$. Identity (5.9) follows directly from the continuity of the inner product $\langle\cdot, \cdot\rangle$ of $\mathcal{H}$ defined on (1.10) above.

LEMMA 5.5. Let $y(x, \lambda)$ be the solution of the initial value problem (1.14), and let (2.1) be valid for $x \in[0, \infty)$ and $\lambda \in \mathbb{C}$. If $\left\{\mu_{k}\right\}_{k=1}^{\infty}$ is a sequence of complex numbers that converges to $\mu \in \mathbb{C}$, then $\left\{y\left(x, \mu_{k}\right)\right\}_{k=1}^{\infty}$ converges uniformly in q-mean to $y(x, \mu)$.

Proof. From Remark 2.2, we conclude that

$$
\lim _{n \rightarrow \infty} y\left(x q^{n}, \lambda\right)=y(0, \lambda), \quad x>0
$$


uniformly on any compact subset of $\mathbb{C}$. Let $x_{0}$ be a positive number. Let $\epsilon>0$. Since the set $\left\{\mu_{k}, \mu, k \in \mathbb{N}\right\}$ is a compact subset of $\mathbb{C}$, then from (5.11), we can choose $n_{0} \in \mathbb{N}$ sufficiently large such that

$$
\begin{aligned}
\left|y\left(x_{0} q^{j}, \mu_{k}\right)-y\left(0, \mu_{k}\right)\right| & <\frac{\epsilon}{\sqrt{2 x_{0} q^{n_{0}}}}, \\
\left|y\left(x_{0} q^{j}, \mu\right)-y(0, \mu)\right| & <\frac{\epsilon}{\sqrt{2 x_{0} q^{n_{0}}}}, \quad j \geq n_{0}
\end{aligned}
$$

for all $k \in \mathbb{N}$. Now

$$
\begin{aligned}
\int_{0}^{x_{0}}\left|y\left(t, \mu_{k}\right)-y(t, \mu)\right|^{2} d_{q} t= & \sum_{j=0}^{n_{0}-1} x_{0} q^{j}(1-q)\left|y\left(x_{0} q^{j}, \mu_{k}\right)-y\left(x_{0} q^{j}, \mu\right)\right|^{2} \\
& +\int_{0}^{x_{0} q^{n_{0}}}\left|y\left(t, \mu_{k}\right)-y(t, \mu)\right|^{2} d_{q} t .
\end{aligned}
$$

But for each fixed $x, y(x, \lambda)$ is entire. Then the limit of the finite sum in (5.13) as $k \rightarrow \infty$ is zero. Therefore, the lemma follows if we prove that the integral on the right-hand side of (5.13) tends to zero as $k \rightarrow \infty$. Since $y(0, \mu)=y\left(0, \mu_{k}\right)$, then from the Minkowski inequality, we have

$$
\begin{aligned}
\left(\int_{0}^{x_{0} q^{n_{0}}}\left|y\left(t, \mu_{k}\right)-y(t, \mu)\right|^{2} d_{q} t\right)^{1 / 2} \leq & \left(\int_{0}^{x_{0} q^{n_{0}}}\left|y\left(t, \mu_{k}\right)-y\left(0, \mu_{k}\right)\right|^{2} d_{q} t\right)^{1 / 2} \\
& +\left(\int_{0}^{x_{0} q^{n_{0}}}|y(t, \mu)-y(0, \mu)|^{2} d_{q} t\right)^{1 / 2} .
\end{aligned}
$$

Consequently, from (5.12), we have

$$
\int_{0}^{x_{0} q^{n_{0}}}\left|y\left(t, \mu_{k}\right)-y(t, \mu)\right|^{2} d_{q} t<\epsilon^{2}, \quad \text { for all } k \in \mathbb{N}
$$

that is,

$$
\lim _{k \rightarrow \infty} \int_{0}^{x_{0} q^{n_{0}}}\left|y\left(t, \mu_{k}\right)-y(t, \mu)\right|^{2}=0,
$$

which proves the lemma.

LEMma 5.6. The functions

$$
\psi_{n}(x)=\left|r_{n}\right|^{\frac{1}{2}} \theta\left(x, \lambda_{n}\right), \quad n \in \mathbb{N}
$$

form an orthonormal set in $\mathcal{H}$, where $r_{n}$ as given by (4.30) is the residue of $m(\lambda)$ at $\lambda_{n}$. 
Proof. Let $n \in \mathbb{N}$ be fixed, and let $\lambda_{n}$ be an eigenvalue. We first assume that $r_{n}>0$. Let $\lambda_{n, k}=\lambda_{n}+i v_{k}, v_{k} \rightarrow 0$ as $k \rightarrow \infty$. Then for any fixed $m \in \mathbb{N}$,

$$
\begin{gathered}
\int_{0}^{b q^{-m}}\left|v_{k} \psi\left(x, \lambda_{n, k}\right)+i r_{n} \theta\left(x, \lambda_{n}\right)\right|^{2} d_{q} x \\
=\int_{0}^{b q^{-m}} \mid v_{k} \phi\left(x, \lambda_{n, k}\right)+\left\{v_{k} m\left(\lambda_{n, k}\right)+i r_{n}\right\} \theta\left(x, \lambda_{n, k}\right) \\
-\left.i r_{n}\left\{\theta\left(x, \lambda_{n, k}\right)-\theta\left(x, \lambda_{n}\right)\right\}\right|^{2} d_{q} x
\end{gathered}
$$

Applying the Minkowski inequality yields

$$
\begin{aligned}
& \left(\int_{0}^{q^{-m}} \quad\left|v_{k} \psi\left(x, \lambda_{n, k}\right)+i r_{n} \theta\left(x, \lambda_{n}\right)\right|^{2} d_{q} x\right)^{1 / 2} \\
& \quad \leq\left|v_{k}\right|\left(\int_{0}^{q^{-m}}\left|\phi\left(x, \lambda_{n, k}\right)\right|^{2} d_{q} x\right)^{1 / 2} \\
& \quad+\left|v_{k} m\left(\lambda_{n, k}\right)+i r_{n}\right|\left(\int_{0}^{q^{-m}}\left|\theta\left(x, \lambda_{n, k}\right)\right|^{2} d_{q} x\right)^{1 / 2} \\
& \quad+\left|r_{n}\right|\left(\int_{0}^{q^{-m}}\left|\theta\left(x, \lambda_{n, k}\right)-\theta\left(x, \lambda_{n}\right)\right|^{2} d_{q} x\right)^{1 / 2}
\end{aligned}
$$

Using the definition of $r_{n}(4.30), v_{k} m\left(\lambda_{n, k}\right)+i r_{n}$ tends to zero as $k \rightarrow \infty$. Also, from Lemma 2.3, we conclude that

$$
\lim _{m \rightarrow \infty} \int_{0}^{q^{-m}}\left|\theta\left(x, \lambda_{n, k}\right)-\theta\left(x, \lambda_{n}\right)\right|^{2} d_{q} x=0
$$

Hence, the sequence $v_{k} \psi\left(x, \lambda_{n, k}\right)$ converges in $q$-mean to the function $-i r_{n} \times$ $\theta\left(x, \lambda_{n}\right)$. Also, by (5.6) and (5.9),

$$
\int_{0}^{\infty}\left|v_{k} \psi\left(x, \lambda_{n, k}\right)\right|^{2} d_{q} x \leq\left|v_{k} m\left(\lambda_{n, k}\right)\right|=O(1)
$$

as $k \rightarrow 0$. Hence, applying Lemma 5.4 yields

$$
\lim _{k \rightarrow \infty} \int_{0}^{\infty} v_{k} \psi\left(x, \lambda_{n, k}\right) g(x) d_{q} x=-i r_{n} \int_{0}^{\infty} \theta\left(x, \lambda_{n}\right) g(x) d_{q} x
$$


for any function $g$ in $\mathcal{H}$. Set $g(x):=\psi(x, \lambda), \Im(\lambda) \neq 0$. Then from (5.5), we have

$$
\begin{aligned}
\int_{0}^{\infty} \theta\left(x, \lambda_{n}\right) \psi(x, \lambda) d_{q} x & =\lim _{k \rightarrow \infty} \frac{i v_{k}}{r_{n}} \int_{0}^{\infty} \psi\left(x, \lambda_{n, k}\right) \psi(x, \lambda) d_{q} x \\
& =\lim _{k \rightarrow \infty} \frac{i v_{k}\left(m\left(\lambda_{n, k}\right)-m(\lambda)\right)}{r_{n}\left(\lambda-\lambda_{n, k}\right)}=\frac{1}{\lambda-\lambda_{n}}
\end{aligned}
$$

Since

$$
\overline{\theta\left(x, \lambda_{m}\right)}=\theta\left(x, \bar{\lambda}_{m}\right)=\theta\left(x, \lambda_{m}\right),
$$

then substituting with $g(x):=\theta\left(x, \lambda_{m}\right)$ in (5.15) and using (5.16) imply that

$$
\begin{aligned}
\int_{0}^{\infty} \theta\left(x, \lambda_{n}\right) \overline{\theta\left(x, \lambda_{m}\right)} d_{q} x & =\lim _{k \rightarrow \infty} \frac{i v_{k}}{r_{n}} \int_{0}^{\infty} \psi\left(x, \lambda_{n, k}\right) \theta\left(x, \lambda_{m}\right) d_{q} x \\
& =\lim _{k \rightarrow \infty} \frac{i v_{k}}{r_{n}\left(\lambda_{n, k}-\lambda_{m}\right)}=0
\end{aligned}
$$

for all $m \neq n$. If in (5.15) we take $g(x)=\overline{\theta\left(x, \lambda_{n}\right)}=\theta\left(x, \lambda_{n}\right)$, then

$$
\int_{0}^{\infty}\left|\theta\left(x, \lambda_{n}\right)\right|^{2} d_{q} x=\lim _{k \rightarrow \infty} \frac{i v_{k}}{r_{n}\left(\lambda_{n, k}-\lambda_{n}\right)}=\frac{1}{r_{n}} .
$$

Hence, we proved the required result in the case when $r_{n}>0$. Similarly, we prove the result in the case when $r_{n}<0$. We redefine the function $\psi(x, \lambda)$ to be

$$
\psi(x, \lambda)=\phi(x, \lambda)-m(\lambda) \theta(x, \lambda)
$$

for any nonreal $\lambda$. In this case, the sequence $v_{k} \psi\left(x, \lambda_{n, k}\right)$ converges in $q$ mean to $-i\left|r_{n}\right| \theta\left(x, \lambda_{n}\right)$. Taking into consideration that according to (5.17), formula (5.5) will be

$$
\int_{0}^{\infty} \psi(x, \lambda) \psi\left(x, \lambda^{\prime}\right) d_{q} x=\frac{m(\lambda)-m\left(\lambda^{\prime}\right)}{\lambda-\lambda^{\prime}} .
$$

TheOREM 5.7. Let $f \in \mathcal{H}$. Then for $\lambda \neq \lambda_{n}$, the function

$$
\Phi(x, \lambda):=\psi(x, \lambda) \int_{0}^{x} \theta(q t, \lambda) f(q t) d_{q} t+\theta(x, \lambda) \int_{x}^{\infty} \psi(q t, \lambda) f(q t) d_{q} t
$$

is the unique solution of the q-initial value problem

$$
\begin{aligned}
L_{x} y(x)-\lambda y(x) & =f(x), \\
\Phi(0, \lambda) \cos \alpha+D_{q^{-1}} \Phi(0, \lambda) \sin \alpha & =0 .
\end{aligned}
$$


Moreover, $\Phi(x, \lambda)$ is a meromorphic function of $\lambda$ with simple poles at $\lambda_{n}$ with the residue $c_{n} \psi_{n}(x)$,

$$
c_{n}:=\left\langle f, \psi_{n}\right\rangle=\int_{0}^{\infty} f(x) \psi_{n}(x) d_{q} x .
$$

Proof. If $\lambda \neq \lambda_{n}$, then

$$
\begin{aligned}
-\frac{1}{q} D_{q^{-1}} D_{q} \Phi(x, \lambda)= & -\frac{1}{q} D_{q^{-1}} D_{q} \psi(x, \lambda) \int_{0}^{x} \theta(q t, \lambda) f(q t) d_{q} t \\
& -\frac{1}{q} D_{q^{-1}} D_{q} \theta(x, \lambda) \int_{x}^{\infty} \psi(q t, \lambda) f(q t) d_{q} t \\
& +\left|\begin{array}{cc}
\psi(x, \lambda) & \theta(x, \lambda) \\
D_{q} \psi\left(q^{-1} x, \lambda\right) & D_{q} \theta\left(q^{-1} x, \lambda\right)
\end{array}\right| f(x) .
\end{aligned}
$$

Using that $g(x)=g\left(q^{-1} x\right)-x q^{-1}(1-q) D_{q} g\left(q^{-1} x\right)$ for any function defined on a $q$-geometric set and that $\psi(x, \lambda)=\phi(x, \lambda)+m(\lambda) \theta(x, \lambda)$, we obtain

$$
\begin{aligned}
\left|\begin{array}{cc}
\psi(x, \lambda) & \theta(x, \lambda) \\
D_{q} \psi\left(q^{-1} x, \lambda\right) & D_{q} \theta\left(q^{-1} x, \lambda\right)
\end{array}\right| & =\left|\begin{array}{cc}
\psi\left(q^{-1} x, \lambda\right) & \theta\left(q^{-1} x, \lambda\right) \\
D_{q} \psi\left(q^{-1} x, \lambda\right) & D_{q} \theta\left(q^{-1} x, \lambda\right)
\end{array}\right| \\
& =W(\psi, \theta)\left(q^{-1} x\right)=W(\phi, \theta)\left(q^{-1} x\right) \\
& =W(\phi, \theta)(0)=1 .
\end{aligned}
$$

Since $\theta(x, \lambda), \psi(x, \lambda)$ are solutions of $(1.14)$, then

$$
-\frac{1}{q} D_{q^{-1}} D_{q} \Phi(x, \lambda)=(u(x)-\lambda) \Phi(x, \lambda)+f(x) .
$$

Also,

$$
\begin{gathered}
\Phi(0, \lambda)=\theta(0, \lambda) \int_{0}^{\infty} \psi(q t, \lambda) f(q t) d_{q} t \\
D_{q^{-1}} \Phi(0, \lambda)=D_{q^{-1}} \theta(0, \lambda) \int_{0}^{\infty} \psi(q t, \lambda) f(q t) d_{q} t
\end{gathered}
$$

then using the initial conditions $(2.6)-(2.7)$, we get

$$
\Phi(0, \lambda) \cos \alpha+D_{q^{-1}} \Phi(0, \lambda) \sin \alpha=0 .
$$


Hence, $\Phi_{X}(x, \lambda)$ is the solution of (5.19). Now we study the analyticity of $\Phi(x, \lambda)$. Let $\Phi_{X}(x, \lambda)$ be the solution of (5.19) corresponding to the functions with $f(y)=0$ for $y>X, X \in\left\{q^{-m}, m \in \mathbb{N}\right\}$. Hence,

$$
\begin{aligned}
\Phi_{X}(x, \lambda)= & \phi(x, \lambda) \int_{0}^{x} \theta(q t, \lambda) f(q t) d_{q} t+\theta(x, \lambda) \int_{x}^{X} \phi(q t, \lambda) f(q t) d_{q} t \\
& +m(\lambda) \theta(x, \lambda) \int_{0}^{X} \theta(q t, \lambda) f(q t) d_{q} t .
\end{aligned}
$$

This is analytic everywhere except at $\lambda=\lambda_{n}, n=0,1,2, \ldots$, where it has simple poles with residues

$$
c_{n, X}:=\lim _{n \rightarrow \infty}\left(\lambda-\lambda_{n}\right) \Phi_{X}(x, \lambda)=r_{n} \theta\left(x, \lambda_{n}\right) \int_{0}^{X} \theta\left(q t, \lambda_{n}\right) f(q t) d_{q} t .
$$

Let $\mathcal{C}$ denote the rectangle

$$
\xi_{1} \leq \Re(z) \leq \xi_{2}, \quad-\eta \leq \Im(z) \leq \eta,
$$

excluding all $\lambda_{n}$. Then by the Cauchy local formula (see [33]), we have

$$
\Phi_{X}(x, \lambda)=\frac{1}{2 \pi i} \int_{\mathcal{C}} \frac{\Phi_{X}(x, z)}{z-\lambda} d z, \quad \lambda \in \operatorname{int} \mathcal{C} .
$$

If $\lambda$ is not real, then $\Phi_{X}(x, \lambda) \rightarrow \Phi(x, \lambda)$ as $X \rightarrow \infty$, and because $v=\Im(\lambda) \rightarrow$ $0, \lambda$ tends to a value that is not an eigenvalue, we obtain by using the Cauchy-Schwarz inequality and (5.6) that

$$
\begin{aligned}
\Phi_{X}(x, \lambda) & =O\left\{\int_{x}^{\infty} \psi(q t, \lambda) f(q t) d_{q} t\right\}=O\left\{\int_{0}^{\infty}|\psi(q t, \lambda)|^{2} d_{q} t\right\}^{\frac{1}{2}} \\
& =O\left(|v|^{-\frac{1}{2}}\right) .
\end{aligned}
$$

Letting $X \rightarrow \infty$ in (5.21) and applying the dominated convergence theorem give

$$
\Phi(x, \lambda)=\frac{1}{2 \pi i} \int_{\mathcal{C}} \frac{\Phi(x, z)}{z-\lambda} d_{q} z .
$$

Hence, $\Phi(x, \lambda)$ is analytic in $\mathcal{C}$; that is, the functions so denoted in the upper and lower half-planes are analytic continuations of each other. If $\mathcal{C}$ includes a point $\lambda_{n}$, then $\Phi(x, \lambda)$ has a simple pole at $\lambda_{n}$, and its residue there is the limit of $c_{n, X}$ as $X \rightarrow \infty$; that is,

$$
r_{n} \theta\left(x, \lambda_{n}\right) \int_{0}^{\infty} \theta\left(q t, \lambda_{n}\right) f(q t) d_{q} t=\psi_{n}(x) \int_{0}^{\infty} \psi_{n}(q t) f(q t) d_{q} t=c_{n} \psi_{n}(x) .
$$


Let $\widetilde{\mathcal{H}}$ be the subspace of $\mathcal{H}$ of all functions $f$ such that

(1) $\tilde{f}(x):=u(x) f(x)-(1 / q) D_{q^{-1}} D_{q} f(x) \in \mathcal{H}$;

(2) $f(0) \cos \alpha+D_{q^{-1}} f(0) \sin \alpha=0$;

(3) $\lim _{n \rightarrow \infty} W_{q}(\psi(\cdot, \lambda), f(\cdot))\left(q^{-n}\right)=0$, for every nonreal $\lambda$.

The set $\widetilde{\mathcal{H}}$ is a subspace of $\mathcal{H}$. The subspace $\widetilde{\mathcal{H}}$ may be characterized using the $q$-analogs of the theories developed by [23] and [25]. However, this needs separate investigation. We first prove the expansion theorem for functions on the space $\widetilde{\mathcal{H}}$; then we prove it for any function on the space $\mathcal{H}$.

Lemma 5.8. Let $\lambda$ be a nonreal complex number. If $f \in \mathcal{H}$, then

$$
\int_{0}^{\infty}|\Phi(x, \lambda)|^{2} d_{q} x \leq \frac{1}{|v|} \int_{0}^{\infty}|f(x)|^{2} d_{q} x, \quad v:=\Im(\lambda) .
$$

Proof. Assume first that $f\left(q^{-k}\right)=0$ for all $k>m, m \in \mathbb{N}$. We denote the corresponding $\Phi(\cdot, \lambda)$ and $f(\cdot)$ by $\Phi_{m}(\cdot, \lambda)$ and $f_{m}(\cdot)$, respectively. Then from (5.18), we have

$$
\begin{aligned}
\Phi_{m}(0, \lambda) & =\sin \alpha \int_{0}^{q^{-m}} \psi(q t, \lambda) f_{m}(q t) d_{q} t \\
D_{q} \Phi_{m}(0, \lambda) & =-\cos \alpha \int_{0}^{q^{-m}} \psi(q t, \lambda) f_{m}(q t) d_{q} t
\end{aligned}
$$

for any nonreal $\lambda$, and

$$
\begin{gathered}
\Phi_{m}\left(q^{-j}, \lambda\right)=\psi\left(q^{-j}, \lambda\right) \int_{0}^{q^{-m}} \theta(q t, \lambda) f_{m}(q t) d_{q} t \\
D_{q} \Phi_{m}\left(q^{-j}, \lambda\right)=D_{q} \psi\left(q^{-j}, \lambda\right) \int_{0}^{q^{-m}} \theta(q t, \lambda) f_{m}(q t) d_{q} t
\end{gathered}
$$

for all $j \geq m$. Consequently,

$$
\begin{aligned}
W_{q}\left(\Phi_{m}(\cdot, \lambda), \Phi_{m}\left(\cdot, \lambda^{\prime}\right)\right)(0)= & 0 \\
W_{q}\left(\Phi_{m}(\cdot, \lambda), \Phi_{m}\left(\cdot, \lambda^{\prime}\right)\right)\left(q^{-j}\right)= & W_{q}\left(\psi(\cdot, \lambda), \psi\left(\cdot, \lambda^{\prime}\right)\right)\left(q^{-j}\right) \\
& \times \int_{0}^{q^{-m}} \theta(q t, \lambda) f_{m}(q t) d_{q} t .
\end{aligned}
$$

From Lemma 5.1, we obtain

$$
\lim _{j \rightarrow \infty} W_{q}\left(\Phi_{m}(\cdot, \lambda), \Phi_{m}\left(\cdot, \lambda^{\prime}\right)\right)\left(q^{-j}\right)=0 .
$$


Substituting with $y(t)=\Phi_{m}(t, \lambda)$ and $z(t)=\Phi_{m}\left(t, \lambda^{\prime}\right)$ in $(2.9)$ gives

$$
\begin{gathered}
\int_{0}^{q^{-j}}\left(\Phi_{m}(t, \lambda) L_{t} \Phi_{m}\left(t, \lambda^{\prime}\right)-\Phi_{m}\left(t, \lambda^{\prime}\right) L_{t} \Phi_{m}(t, \lambda)\right) d_{q} t \\
=-W_{q}\left(\Phi_{m}(\cdot, \lambda), \Phi_{m}\left(\cdot, \lambda^{\prime}\right)\right)\left(q^{-j-1}\right) .
\end{gathered}
$$

Letting $j \longrightarrow \infty$ in the above equation yields

$$
\int_{0}^{\infty} \Phi_{m}(t, \lambda) L_{t} \Phi_{m}\left(t, \lambda^{\prime}\right) d_{q} t=\int_{0}^{\infty} \Phi_{m}\left(t, \lambda^{\prime}\right) L_{t} \Phi_{m}(t, \lambda) d_{q} t .
$$

If $\lambda^{\prime}=\bar{\lambda}$, then

$\int_{0}^{\infty} \Phi_{m}(t, \lambda)\left\{\bar{\lambda} \Phi_{m}(t, \bar{\lambda})-f_{m}(t)\right\} d_{q} t=\int_{0}^{\infty} \Phi_{m}(t, \bar{\lambda})\left\{\lambda \Phi_{m}(t, \lambda)-f_{m}(t)\right\} d_{q} t ;$

that is,

$$
(\lambda-\bar{\lambda}) \int_{0}^{\infty}\left|\Phi_{m}(t, \lambda)\right|^{2} d_{q} t=\int_{0}^{\infty}\left\{\Phi_{m}(t, \bar{\lambda})-\Phi_{m}(t, \lambda)\right\} f_{m}(t) d_{q} t .
$$

Hence, if $\lambda=\mu+i v, v>0$, then

$$
\begin{aligned}
2 v \int_{0}^{\infty}\left|\Phi_{m}(t, \lambda)\right|^{2} d_{q} t & \leq 2 \int_{0}^{\infty}\left|\Phi_{m}(t, \lambda) f_{m}(t)\right| d_{q} t \\
& \leq 2\left\{\int_{0}^{\infty}\left|\Phi_{m}(t, \lambda)\right|^{2} d_{q} t \int_{0}^{\infty}\left|f_{m}(t)\right|^{2} d_{q} t\right\}^{\frac{1}{2}} .
\end{aligned}
$$

That is,

$$
\int_{0}^{\infty}\left|\Phi_{m}(t, \lambda)\right|^{2} d_{q} t \leq \frac{1}{v^{2}} \int_{0}^{\infty}\left|f_{m}(t)\right|^{2} d_{q} t
$$

which is the required result for the terminated $f$. Now if $f$ is any function in $\mathcal{H}$, then for fixed $n \in \mathbb{N}$, we get

$$
\lim _{m \rightarrow \infty} \int_{0}^{q^{-n}}\left|\Phi_{m}(t, \lambda)\right|^{2} d_{q} t=\int_{0}^{q^{-n}}|\Phi(t, \lambda)|^{2} d_{q} t .
$$

But

$$
\begin{aligned}
\int_{0}^{q^{-n}}\left|\Phi_{m}(t, \lambda)\right|^{2} d_{q} t & \leq \int_{0}^{\infty}\left|\Phi_{m}(t, \lambda)\right|^{2} d_{q} t \leq \frac{1}{v^{2}} \int_{0}^{\infty}\left|f_{m}(t)\right|^{2} d_{q} t \\
& =\frac{1}{v^{2}} \int_{0}^{q^{-m}}|f(t)|^{2} d_{q} t \leq \frac{1}{v^{2}} \int_{0}^{\infty}|f(t)|^{2} d_{q} t .
\end{aligned}
$$

The result therefore follows by making $m \rightarrow \infty$ and then letting $n \rightarrow \infty$. 
LEMMA 5.9. If $f \in \widetilde{\mathcal{H}}$ and if $\Phi(x, \lambda)$, defined by (5.18), is also denoted by $\Phi(x, \lambda, f)$, then

$$
\Phi\left(q^{n}, \lambda, f\right)=\frac{1}{\lambda}\left\{f\left(q^{n}\right)+\Phi\left(q^{n}, \lambda, \widetilde{f}\right)\right\}, \quad n \in \mathbb{Z} .
$$

Proof. We have for any $x \in\left\{q^{-n}, n \in \mathbb{Z}\right\}$,

$$
\int_{0}^{x} \theta(q t, \lambda) f(q t) d_{q} t=\frac{1}{\lambda} \int_{0}^{x}\left(u(q t) \theta(q t, \lambda)-\frac{1}{q} D_{q}^{2} \theta(t, \lambda)\right) f(q t) d_{q} t .
$$

Using $q$-integration by parts, we have

$$
\begin{aligned}
\int_{0}^{x} D_{q}^{2} \theta(t, \lambda) f(q t) d_{q} t= & D_{q} \theta(x, \lambda) f(x)-D_{q} \theta(0, \lambda) f(0) \\
& -\int_{0}^{x} D_{q} \theta(t, \lambda) D_{q} f(t) d_{q} t \\
\int_{0}^{x} D_{q} \theta(t, \lambda) D_{q} f(t) d_{q} t= & \theta(x, \lambda) D_{q} f(x)-\theta(0, \lambda) D_{q} f(0) \\
& -\int_{0}^{x} \theta(q t, \lambda) D_{q}^{2} f(t) d_{q} t .
\end{aligned}
$$

Hence,

$$
\int_{0}^{x} D_{q}^{2} \theta(t, \lambda) f(q t) d_{q} t=-\left[W_{q}(\theta(\cdot, \lambda), f(\cdot))(t)\right]_{0}^{x}-\int_{0}^{x} \theta(t, \lambda) D_{q}^{2} f(t) d_{q} t
$$

Since $f \in \widetilde{\mathcal{H}}$, then

$$
W_{q}(\theta(\cdot, \lambda), f(\cdot))(0)=W_{q}(\psi(\cdot, \lambda), f(\cdot))(0)=0
$$

Therefore,

$$
\begin{aligned}
\int_{0}^{x} \theta(q t, \lambda) f(q t) d_{q} t= & \frac{1}{\lambda} W_{q}(\theta(\cdot, \lambda), f)(x) \\
& +\frac{1}{\lambda} \int_{0}^{x}\left(u(q t) f(q t)-\frac{1}{q} D_{q}^{2} f(t)\right) \theta(q t, \lambda) d_{q} t \\
= & \frac{1}{\lambda} W_{q}(\theta(\cdot, \lambda), f)(x)+\frac{1}{\lambda} \int_{0}^{x} \widetilde{f}(q t) \theta(q t, \lambda) d_{q} t
\end{aligned}
$$


Similarly,

$$
\begin{aligned}
\int_{x}^{\infty} \psi(q t, \lambda) f(q t) d_{q} t= & \frac{1}{\lambda}\left[\lim _{j \rightarrow \infty} W_{q}(\psi(\cdot, \lambda), f)\left(q^{j}\right)-W_{q}(\psi(\cdot, \lambda), f)(x)\right] \\
& +\frac{1}{\lambda} \int_{x}^{\infty} \widetilde{f}(q t) \psi(q t, \lambda) d_{q} t .
\end{aligned}
$$

But $\lim _{j \rightarrow \infty} W_{q}(\psi(\cdot, \lambda), f)\left(b q^{j}\right)=0$. Hence,

$$
\begin{aligned}
\int_{x}^{\infty} \psi(q t, \lambda) f(q t) d_{q} t= & -\frac{1}{\lambda} W_{q}(\psi(\cdot, \lambda), f)(x) \\
& +\frac{1}{\lambda} \int_{x}^{\infty} \widetilde{f}(q t) \psi(q t, \lambda) d_{q} t
\end{aligned}
$$

Substituting from (5.23) and (5.24) in (5.18) gives

$$
\begin{aligned}
\Phi(x, \lambda, f)= & \frac{1}{\lambda}\left(\psi(x, \lambda) W_{q}(\theta(\cdot, \lambda), f)(x)-\theta(x, \lambda) W_{q}(\psi(\cdot, \lambda), f)(x)\right) \\
& +\frac{1}{\lambda} \Phi(x, \lambda, \tilde{f}) .
\end{aligned}
$$

Noting that

$$
\begin{aligned}
& \psi(x, \lambda) W_{q}(\theta(\cdot, \lambda), f)(x)-\theta(x, \lambda) W_{q}(\psi(\cdot, \lambda), f)(x) \\
& \quad=f(x) W_{q}(\theta(\cdot, \lambda), \phi(\cdot, \lambda))(x)=f(x) W_{q}(\theta(\cdot, \lambda), \phi(\cdot, \lambda))(0)=f(x),
\end{aligned}
$$

then (5.25) is the required identity and the lemma follows.

REMARK 5.10. Mainly if $\lambda$ is not an eigenvalue of (1.14) and $\Phi(x, \lambda)$ is a solution of the boundary value problem (5.19), where $f \in \mathcal{H}$, then

$$
\Phi(x, \lambda, f)=\int_{0}^{\infty} G(x, q y, \lambda) f(q y) d_{q} y, \quad x \in\left\{0, q^{-n}, n \in \mathbb{N}\right\},
$$

where $G(x, y, \lambda)$ is Green's function of (5.19). So in our notation

$$
G(x, y, \lambda)= \begin{cases}\psi(x, \lambda) \theta(y, \lambda), & 0 \leq y \leq x<\infty \\ \theta(x, \lambda) \psi(y, \lambda), & 0 \leq x \leq y<\infty\end{cases}
$$

Also, we can restate

$$
\Phi(x, \lambda, \widetilde{f})=\int_{0}^{\infty} G(x, q y, \lambda) \widetilde{f}(q y) d_{q} y, \quad x \in\left\{0, q^{-n}, n \in \mathbb{N}\right\}
$$


Then (5.22) is

$$
f(x)=\int_{0}^{\infty} G(x, q y, \lambda)(\widetilde{f}(q y)-\lambda f(q y)) d_{q} y, \quad x \in\left\{q^{m}, m \in \mathbb{Z}\right\} .
$$

Bessel's inequality implies that if $f \in \mathcal{H}$, then

$$
\sum_{n=0}^{\infty} c_{n}^{2} \leq \int_{0}^{\infty}|f(y)|^{2} d_{q} y, \quad c_{n}=\left\langle f, \psi_{n}\right\rangle
$$

LEMMA 5.11. Let $F(\lambda)$ be an analytic function of $\lambda=\mu+i v$, regular for $-r \leq \mu \leq r,-r \leq v \leq r$, and let

$$
|F(\lambda)| \leq \frac{M}{|v|}
$$

in this square. Then

$$
|F(\lambda)| \leq \frac{3 M}{r} \quad(\mu=0,-r \leq v \leq r)
$$

Proof. See [47, p. 35].

In the following we prove the expansion theorems for all functions in $\widetilde{\mathcal{H}}$. Since any complex-valued function $f$ has the representation $f=f_{1}+i f_{2}$, where $f_{1}$ and $f_{2}$ are real-valued functions, we prove the expansion theorems under the assumption that $\widetilde{\mathcal{H}}$ is real, and the results when $\widetilde{\mathcal{H}}$ is complex follow directly.

THEOREM 5.12. If $f \in \tilde{\mathcal{H}}$, then

$$
\int_{0}^{\infty}|f(x)|^{2} d_{q} x=\sum_{n=0}^{\infty}\left|c_{n}\right|^{2}
$$

Proof. Let $f \in \widetilde{\mathcal{H}}$, and assume that $f(x)=0$ for sufficiently large values of $x$. Let

$$
\Psi(\lambda)=\int_{0}^{\infty} f(x) \Phi(x, \lambda) d_{q} x .
$$

Then $\Psi(\lambda)$ is regular except for simple poles at the points $\lambda_{n}$, where it has residues

$$
c_{n} \int_{0}^{\infty} f(x) \psi_{n}(x, \lambda) d_{q} x=c_{n}^{2} .
$$


By (5.22), we have

$$
\Psi(\lambda)=\frac{1}{\lambda} \int_{0}^{\infty}|f(x)|^{2} d_{q} x+\frac{1}{\lambda} \int_{0}^{\infty} \Phi(x, \lambda, \tilde{f}) f(x) d_{q} x .
$$

Applying Lemma 5.8 to $\tilde{f}$ yields

$$
\begin{aligned}
\left|\frac{1}{\lambda} \int_{0}^{\infty} \Phi(x, \lambda, \tilde{f}) f(x) d_{q} x\right| & \leq \frac{1}{|\lambda|}\left(\int_{0}^{\infty}|\Phi(x, \lambda, \widetilde{f})|^{2} d_{q} x \int_{0}^{\infty}|f(x)|^{2} d_{q} x\right)^{\frac{1}{2}} \\
& \leq \frac{1}{|\lambda v|}\|f\|^{2} .
\end{aligned}
$$

Let $\mathcal{C}(R)$ denote the contour formed by the segments of lines $(R-i, R+i)$ and $(-R-i,-R+i)$, joined by the semicircles of radius $R$ and centers $\pm i$. Then

$$
\int_{\mathcal{C}(R)} \Psi(\lambda) d \lambda=2 \pi i \sum_{-R \leq \lambda_{n} \leq R} c_{n}^{2}
$$

if none of the $\lambda_{n}$ equals $\pm R$. On the upper semicircle in the first quadrant, we have

$$
\lambda=i+R \exp (i \varphi) \quad\left(0 \leq \varphi \leq \frac{1}{2} \pi\right) .
$$

We choose $R$ sufficiently large such that

$$
R \frac{\pi}{2}>1, \quad 1+R-R \frac{\pi}{2}>0
$$

Hence,

$$
1+R \sin \varphi>R \varphi, \quad \varphi \in\left[\frac{1}{R}, \frac{\pi}{2}\right]
$$

Hence, the last term in (5.27), integrated around the quadrant defined in (5.28), satisfies

$$
\begin{aligned}
O\left(\int_{0}^{\frac{1}{2} \pi} \frac{R \varphi}{R(1+R \sin \varphi)}\right) & =O\left(\int_{0}^{\frac{1}{R}} d \varphi\right)+O\left(\int_{\frac{1}{R}}^{\frac{1}{2} \pi} \frac{d \varphi}{R \varphi}\right) \\
& =O\left(\frac{1}{R}\right)+O\left(\frac{\log R}{R}\right)=o(1)
\end{aligned}
$$

as $R \rightarrow \infty$. A similar argument applies for the other quadrants. Hence, the integral of $\Psi(\lambda)$ along each semicircle tends to

$$
\pi i \int_{0}^{\infty}|f(x)|^{2} d_{q} x
$$


as $R \rightarrow \infty$. To prove the theorem, it suffices to show that

$$
\lim _{R \rightarrow \infty} \int_{R-i}^{R+i} \Psi(\lambda) d \lambda=0, \quad \lim _{R \rightarrow \infty} \int_{-R-i}^{-R+i} \Psi(\lambda) d \lambda=0 .
$$

Let

$$
\chi(\lambda)=\Psi(\lambda)-\sum_{R-1 \leq \lambda_{n} \leq R+1} \frac{c_{n}^{2}}{\lambda-\lambda_{n}} .
$$

Then $\chi(\lambda)$ is regular for $R-1 \leq \lambda \leq R+1$, and

$$
|\chi(\lambda)|<\frac{K}{|\lambda v|}+\frac{1}{|v|} \sum_{R-1 \leq \lambda_{n} \leq R+1} c_{n}^{2} \leq \frac{\epsilon(R)}{|v|},
$$

where $\epsilon(R) \rightarrow 0$ as $R \rightarrow \infty$. Therefore, by Lemma 5.11 ,

$$
|\chi(\lambda)| \leq 3 \epsilon(R)
$$

on the segment $(R-i, R+i)$. Hence,

$$
\lim _{R \rightarrow \infty} \int_{R-i}^{R+i} \chi(\lambda) d \lambda=0 .
$$

Also, since the path of integration can be replaced by a semicircle on the side opposite to $\lambda_{n}$, and on this semicircle the integrand is bounded, then

$$
\int_{R-i}^{R+i} \frac{d \lambda}{\lambda-\lambda_{n}}=O(1), \quad \text { as } R \rightarrow \infty
$$

Therefore,

$$
\int_{R-i}^{R+i} \sum_{R-1 \leq \lambda_{n} \leq R+1} \frac{c_{n}^{2}}{\lambda-\lambda_{n}} d \lambda=O\left(\sum_{R-1 \leq \lambda_{n} \leq R+1} c_{n}^{2}\right)=o(1)
$$

as $R \rightarrow \infty$. Thus, (5.29) is proved for the first integral. The second is similar, and the theorem follows.

THEOREM 5.13. If $f \in \widetilde{\mathcal{H}}$, then

$$
f(x)=\sum_{n=0}^{\infty} c_{n} \psi_{n}(x), \quad x \in\left\{q^{m}, m \in \mathbb{Z}\right\}
$$

is absolutely and uniformly convergent in any finite subset of $\left\{q^{m}, m \in \mathbb{Z}\right\}$. 
Proof. Let $m \in \mathbb{N}$. From Green's identity (2.9), we obtain

$$
\begin{aligned}
& \left(\lambda_{n}-\lambda\right) \int_{0}^{q^{-m}} \psi(x, \lambda) \theta\left(x, \lambda_{n}\right) d_{q} x \\
& \quad=W_{q}\left(\psi(\cdot, \lambda), \theta\left(\cdot, \lambda_{n}\right)\right)(0)-W_{q}\left(\psi(\cdot, \lambda), \theta\left(\cdot, \lambda_{n}\right)\right)\left(q^{-m-1}\right) .
\end{aligned}
$$

From (5.16), we have

$$
\left(\lambda_{n}-\lambda\right) \int_{0}^{\infty} \psi(x, \lambda) \theta\left(x, \lambda_{n}\right) d_{q} x=1 .
$$

Also,

$$
W_{q}\left(\psi(\cdot, \lambda), \theta\left(\cdot, \lambda_{n}\right)\right)(0)=W_{q}\left(\phi(\cdot, \lambda), \theta\left(\cdot, \lambda_{n}\right)\right)(0)=1
$$

Then

$$
\lim _{m \rightarrow \infty} W_{q}\left(\psi(\cdot, \lambda), \theta\left(\cdot, \lambda_{n}\right)\right)\left(q^{-m-1}\right)=0 .
$$

Substituting with $f=\psi_{n}$ in $(5.26)$ and noting that $\widetilde{\psi}_{n}(y)=\lambda_{n} \psi_{n}(y)$, we obtain

$$
\int_{0}^{\infty} G(x, y, \lambda) \psi_{n}(y) d_{q} y=\frac{\psi_{n}(x)}{\lambda_{n}-\lambda} .
$$

Also, for fixed $x=q^{-m_{0}}, m_{0} \in \mathbb{N}$ and fixed nonreal $\lambda$, we have

$$
\begin{aligned}
\int_{0}^{\infty}|G(x, t, \lambda)|^{2} d_{q} t= & |\psi(x, \lambda)|^{2} \int_{0}^{x}\left|\theta\left(t, \lambda_{n}\right)\right|^{2} d_{q} t \\
& +\left|\theta\left(x, \lambda_{n}\right)\right|^{2} \int_{x}^{\infty}|\psi(y, \lambda)|^{2} d_{q} y \leq K .
\end{aligned}
$$

Hence, from Bessel's inequality,

$$
\sum_{n=0}^{\infty}\left|\frac{\psi_{n}(x)}{\lambda_{n}-\lambda}\right|^{2} \leq K
$$

Let $f \in \widetilde{\mathcal{H}}$ be a real-valued function, and let $\lambda \in \mathbb{C}$. Define a function $g$ on $[0, \infty[$ by

$$
g(t, \lambda)=\widetilde{f}(t)-\lambda f(t)
$$

For $k \in \mathbb{N}$, set $\lambda_{n, k}=\lambda_{n}+i v_{k}$, where $v_{k} \rightarrow 0$ as $k \rightarrow \infty$. Since

$$
L_{x} \psi\left(x, \lambda_{n, k}\right)=\lambda_{n, k} \psi\left(x, \lambda_{n, k}\right)
$$


then

$$
\begin{aligned}
\int_{0}^{\infty} \psi\left(t, \lambda_{n, k}\right) g\left(t, \lambda_{n, k}\right) d_{q} t & =\int_{0}^{\infty}\left(\psi(t, \lambda) \widetilde{f}(t)-f(t) L_{t} \psi(t, \lambda)\right) d_{q} t \\
& =\lim _{m \rightarrow \infty} W_{q}(f(\cdot), \psi(\cdot, \lambda))\left(q^{-m}\right)-W_{q}(f, \psi)(0) \\
& =D_{q} f(0) \cos \alpha-f(0) \sin \alpha=0
\end{aligned}
$$

for all $k \in \mathbb{N}$. Similar to Lemma 5.6 ,

$$
\left\|i v_{k} \psi\left(\cdot, \lambda_{n, k}\right)-r_{n} \theta_{n}(\cdot)\right\|, \quad\left\|g\left(\cdot, \lambda_{n, k}\right)-g\left(\cdot, \lambda_{n}\right)\right\|
$$

converge to zero as $k \rightarrow \infty$. Hence, from the continuity of the inner product, we obtain

$$
\lim _{k \rightarrow \infty}\left\langle i v_{k} \psi\left(\cdot, \lambda_{n, k}\right), g\left(\cdot, \lambda_{n, k}\right)\right\rangle=r_{n}\left\langle\theta_{n}(\cdot), g\left(\cdot, \lambda_{n}\right)\right\rangle .
$$

So from (5.32), we obtain $\left\langle\psi_{n}(\cdot), g\left(\cdot, \lambda_{n}\right)\right\rangle=0$. Consequently,

$$
\int_{0}^{\infty} \widetilde{f}(t) \psi_{n}(t) d_{q} t=\lambda_{n} \int_{0}^{\infty} f(t) \psi_{n}(t) d_{q} t=\lambda_{n} c_{n}
$$

Hence, if $d_{n}$ is the Fourier coefficient of $g(x)$, then

$$
\begin{aligned}
d_{n} & =\int_{0}^{\infty} g(x) \psi_{n}(x) d_{q} x=\int_{0}^{\infty} \tilde{f}(x) \psi_{n}(x) d_{q}-\lambda \int_{0}^{\infty} f(x) \psi_{n}(x) d_{q} x \\
& =\left(\lambda_{n}-\lambda\right) c_{n},
\end{aligned}
$$

and

$$
\sum\left|\lambda_{n}-\lambda\right|^{2} c_{n}^{2}
$$

is convergent. From (5.26), it follows that

$$
\begin{aligned}
f(x) & =\int_{0}^{\infty} G(x, y, \lambda) g(y, \lambda) d_{q} y=\langle G(x, \cdot, \lambda), g(\cdot, \lambda)\rangle \\
& =\frac{1}{4}\left(\|G(x, \cdot, \lambda)+g(\cdot, \lambda)\|^{2}+\|G(x, \cdot, \lambda)-g(\cdot, \lambda)\|^{2}\right) \\
& =\frac{1}{4}\left(\sum_{n=0}^{\infty}\left|c_{n}+d_{n}\right|^{2}+\sum_{n=0}^{\infty}\left|c_{n}-d_{n}\right|^{2}\right)=\sum_{n=0}^{\infty} c_{n} d_{n} \\
& =\sum_{n=0}^{\infty} \frac{\psi_{n}(x)}{\lambda_{n}-\lambda}\left(\lambda_{n}-\lambda\right) c_{n}=\sum_{n=0}^{\infty} c_{n} \psi_{n}(x)
\end{aligned}
$$


for all $x \in\left\{q^{m}, m \in \mathbb{Z}\right\}$. The absolute and uniform convergence of the series on any finite subset of $\left\{q^{m}, m \in \mathbb{Z}\right\}$ follows from (5.31) and the convergence of (5.33).

Theorems 5.12 and 5.13 are proved for the proper subspace $\widetilde{\mathcal{H}}$ of $\mathcal{H}$. They can be extended to all functions in $\mathcal{H}$.

Lemma 5.14. The space $\widetilde{\mathcal{H}}$ is a dense subspace of $\mathcal{H}$.

Proof. Let $f \in \mathcal{H}$, and let $\epsilon$ be a positive number. Let $n \in \mathbb{Z}$, and let $\varphi_{n}$ be defined on $[0, \infty)$ by

$$
\varphi_{n}(x)= \begin{cases}\frac{q^{-n / 2}}{\sqrt{1-q}}, & x=q^{n} \\ 0, & \text { otherwise. }\end{cases}
$$

Obviously, $\varphi_{n}(0)=D_{q} \varphi_{n}(0)=1$, and

$$
-\frac{1}{q} D_{q^{-1}} D_{q} \varphi_{n}(x)+u(x) \varphi_{n}(x)= \begin{cases}\frac{q^{-n / 2}}{\sqrt{1-q}} u\left(q^{n}\right), & x=q^{n} \\ 0, & \text { otherwise }\end{cases}
$$

is an $\mathcal{H}$ - function. Moreover, since $W_{q}\left(\psi, \varphi_{n}\right)\left(q^{-m}\right)=0$ for all $m \neq n$, then

$$
\lim _{m \rightarrow \infty} W_{q}\left(\psi, \varphi_{n}\right)\left(q^{-m}\right)=0, \quad \text { for all } n \in \mathbb{Z} .
$$

Hence, $\varphi_{n} \in \widetilde{\mathcal{H}}$. Proving that $\varphi_{n}$ is an orthonormal basis completes the proof. Indeed, for all $n \in \mathbb{Z}$, clearly

$$
\int_{0}^{\infty} \varphi_{n}(t) \varphi_{m}(t)=\delta_{n m}
$$

where $\delta_{n m}$ is the Kronecker delta. To prove the completeness of $\left\{\varphi_{n}: n \in \mathbb{Z}\right\}$ in $\mathcal{H}$, it suffices to prove that the only element in $\mathcal{H}$ which is orthogonal on $\left\{\varphi_{n}: n \in \mathbb{Z}\right\}$ is the zero element. So assume that there exists $f \in \mathcal{H}$ such that

$$
\int_{0}^{\infty} f(t) \varphi_{n}(t) d_{q}(t)=0, \quad \text { for all } n \in \mathbb{Z} .
$$

Hence, $q^{n / 2}(1-q)^{1 / 2} f\left(q^{n}\right)=0$ for all $n \in \mathbb{Z}$. Hence, $f\left(q^{n}\right)=0$ for all $n \in \mathbb{Z}$, and the lemma follows.

Consequently, by the use of the triangle inequality, we obtain the following eigenfunctions expansion theorem. 
THEOREM 5.15. If $f \in \mathcal{H}$, then

$$
f(x)=\sum_{n=0}^{\infty} c_{n} \psi_{n}(x)
$$

with convergence in the $\mathcal{H}$-norm. Moreover, Praseval's equality

$$
\|f\|^{2}=\sum_{n=0}^{\infty}\left|c_{n}\right|^{2}
$$

holds.

\section{§6. q-Bessel problems}

In the following example, the function $u$ has a singularity at $x=0$, so the classification of the singular point is the same as the case of the classification of the singularity at $\infty$. But here $m(\lambda)$ would be the limit of $l_{a}(\lambda)$ as $a \rightarrow 0$. The Hilbert space of consideration here is the space $L_{q}^{2}(0, b)$, where $b$ is a fixed positive number.

Consider the second-order $q$-difference equation

$$
D_{q}^{2} y(x)+\frac{1}{(1-q)^{2}}\left(q^{1-\nu} s^{2}-q^{-1} \frac{\left(1-q^{\nu+\frac{1}{2}}\right)\left(1-q^{\nu-\frac{1}{2}}\right)}{x^{2}}\right) y(q x)=0 .
$$

A fundamental set of solutions of this equation is

$$
y_{1}(x)=x^{\frac{1}{2}} J_{\nu}^{(3)}\left(s x ; q^{2}\right), \quad y_{2}(x)=x^{\frac{1}{2}} Y_{\nu}\left(s x ; q^{2}\right),
$$

where $s:=\sqrt{\lambda}$ is defined with respect to the principal branch and where $Y_{\nu}(x ; q)$ is the function defined for $\nu \notin \mathbb{Z}$ by

$$
Y_{\nu}(x ; q)=\frac{\Gamma_{q}(\nu) \Gamma_{q}(1-\nu)}{\pi}\left\{q^{\nu / 2} \cos \pi \nu J_{\nu}^{(3)}(x ; q)-J_{-\nu}^{(3)}\left(x q^{-\nu / 2} ; q\right)\right\},
$$

and for $n \in \mathbb{Z}$

$$
Y_{n}(x ; q)=\lim _{\nu \rightarrow n} Y_{\nu}(x ; q) .
$$

The function $J_{\nu}^{(3)}(\cdot ; q)$ is the third Jackson $q$-Bessel function defined by

$$
J_{\nu}^{(3)}(x ; q):=x^{\nu} \frac{\left(q^{\nu+1} ; q\right)_{\infty}}{(q ; q)_{\infty}} \sum_{n=0}^{\infty}(-1)^{n} \frac{q^{\frac{n(n+1)}{2}}}{(q ; q)_{n}\left(q^{\nu+1} ; q\right)_{n}} x^{2 n}, \quad \nu>-1
$$


(see [26], [29]). This function is called in some literature the Hahn-Exton $q$-Bessel function (see [44]). Also, we have (see [31], [45])

$$
W_{q}\left(J_{\nu}\left(\cdot ; q^{2}\right), Y_{\nu}\left(\cdot ; q^{2}\right)\right)(x) \equiv \frac{q^{\nu(\nu-1)}(1+q)}{\pi x}, \quad x \in \mathbb{R} \backslash\{0\}
$$

Since the other types of $q$-Bessel functions, that is, $J_{\nu}^{(1)}\left(\cdot ; q^{2}\right), J_{\nu}^{(2)}\left(\cdot ; q^{2}\right)$ (see [26]), will not be used here, from now on we will use the notation $J_{\nu}(\cdot)$ for $J_{\nu}^{(3)}\left(\cdot ; q^{2}\right)$ and $Y_{\nu}(\cdot)$ for $Y_{\nu}\left(\cdot ; q^{2}\right)$. Let $\theta(x, \lambda), \phi(x, \lambda)$ be the solutions which satisfy

$$
\begin{array}{ll}
\phi(b, \lambda)=1, \quad D_{q} \phi(b, \lambda)=0 ; \\
\theta(b, \lambda)=0, \quad D_{q} \theta(b, \lambda)=-1 .
\end{array}
$$

Hence,

$$
\begin{aligned}
& \phi(x, \lambda)=c_{1} x^{\frac{1}{2}} s\left\{J_{\nu}(s x) D_{q} Y_{\nu}(s b)-Y_{\nu}(s x) D_{q} J_{\nu}(s b)\right\}+\frac{\theta(x, \lambda)}{(1+\sqrt{q}) b} \\
& \theta(x, \lambda)=c_{1} q^{-\frac{1}{2}} x^{\frac{1}{2}}\left\{J_{\nu}(s x) Y_{\nu}(s b)-Y_{\nu}(s x) J_{\nu}(s b)\right\}
\end{aligned}
$$

where $c_{1}:=\pi q^{-\nu(\nu-1)} \sqrt{b} /(1+q)$. If $\nu \geq 1$, the only solution of $L_{q}^{2}(0, b)$ is $x^{1 / 2} J_{\nu}(x s)$. So if we seek a solution in $L_{q}^{2}(0, b)$ of the form $\psi(x, \lambda)=\phi(x, \lambda)+$ $m(\lambda) \theta(x, \lambda)$, we should have

$$
m(\lambda)=-s q^{\frac{1}{2}} \frac{D_{q} J_{\nu}(s b)}{J_{\nu}(s b)}-\frac{1}{(1+\sqrt{q}) b} .
$$

The eigenvalues $\lambda_{n}$ are the zeros of $J_{\nu}(s b)$. According to [31], these zeros are real, denumerable, and simple (see also [4], [16], [26]). Now

$$
\lim _{\lambda \rightarrow \lambda_{n}}\left(\lambda-\lambda_{n}\right)^{-1} J_{\nu}(b \sqrt{\lambda})=\frac{b}{2 \sqrt{\lambda_{n}}} J_{\nu}^{\prime}\left(b \sqrt{\lambda_{n}}\right) .
$$

Hence, $m(\lambda)$ has a simple pole at $\lambda_{n}$ with residue $r_{n}$,

$$
r_{n}=\lim _{n \rightarrow \infty}\left(\lambda-\lambda_{n}\right) m(\lambda)=-2 \frac{q^{\frac{1}{2}} \lambda_{n}}{b} \frac{D_{q} J_{\nu}\left(b s_{n}\right)}{J_{\nu}^{\prime}\left(b s_{n}\right)} .
$$

If $\lambda=\lambda_{n}=s_{n}^{2}$, then from $(6.3)$

$$
\theta\left(x, \lambda_{n}\right)=q^{-\frac{1}{2}} c_{1} x^{\frac{1}{2}} Y_{\nu}\left(s_{n} b\right) J_{\nu}\left(s_{n} x\right),
$$


and the normalized eigenfunction is

$$
\psi_{n}:=\left|r_{n}\right|^{\frac{1}{2}} \theta\left(\cdot, \lambda_{n}\right)
$$

Consequently, the Fourier-Bessel expansion is given by

$$
\begin{aligned}
f(x) & =\sum_{n=1}^{\infty}\left\langle f, \psi_{n}\right\rangle \psi_{n}(x) \\
& =\frac{2 c_{1}^{2}}{\sqrt{q} b} x^{\frac{1}{2}} \sum_{n=1}^{\infty}\left|\lambda_{n} \frac{D_{q} J_{\nu}\left(b s_{n}\right) Y_{\nu}^{2}\left(b s_{n}\right)}{J_{\nu}^{\prime}\left(b s_{n}\right)}\right| J_{\nu}\left(s_{n} x\right) \int_{0}^{b} t^{\frac{1}{2}} \overline{J_{\nu}\left(s_{n} t\right)} f(t) d_{q} t .
\end{aligned}
$$

If $\nu= \pm 1 / 2$ there is no singularity. If $0 \leq \nu<1, \nu \neq 1 / 2$, all solutions of (6.1) belong to $L_{q}^{2}(0, b)$, and we are in the limit-circle case, as we will see below. Indeed, from $(6.2)$, the functions $\theta(x, \lambda), \phi(x, \lambda)$ can be written as

$$
\begin{aligned}
\theta(x, \lambda)= & -c_{1} c_{2} q^{-\frac{1}{2}} x^{\frac{1}{2}}\left\{J_{\nu}(s x) J_{-\nu}\left(b s q^{-\nu / 2}\right)-J_{-\nu}\left(s x q^{-\nu / 2}\right) J_{\nu}(b s)\right\}, \\
\phi(x, \lambda)= & -c_{1} c_{2} x^{\frac{1}{2}} s\left\{J_{\nu}(s x) D_{q} J_{-\nu}\left(b s q^{-\nu / 2}\right)-J_{-\nu}\left(s x q^{-\nu / 2}\right) D_{q} J_{\nu}(b s)\right\} \\
& +\frac{\theta(x, \lambda)}{(1+\sqrt{q}) b},
\end{aligned}
$$

where $c_{2}=\left(\Gamma_{q^{2}}(\nu) \Gamma_{q^{2}}(1-\nu)\right) / \pi$. The limit-circle is the limit of the circles

$$
l_{a}(\lambda)=-\frac{\phi(a, \lambda) \cot \beta+D_{q} \phi(a, \lambda)}{\theta(a, \lambda) \cot \beta+D_{q} \theta(a, \lambda)}
$$

as $a \rightarrow 0$. Now for each fixed $s$, we obtain as $x \rightarrow 0$ that

$$
\begin{aligned}
J_{\nu}(s x) & =\frac{\left(q^{2 \nu+2} ; q^{2}\right)_{\infty}}{\left(q^{2} ; q^{2}\right)_{\infty}}(x s)^{\nu}+O\left(x^{\nu+2}\right), \\
D_{q} J_{\nu}(s x) & =\frac{\left(q^{2 \nu+2} ; q^{2}\right)_{\infty}}{\left(q^{2} ; q^{2}\right)_{\infty}} s \frac{1-q^{\nu}}{1-q}(x s)^{\nu-1}+O\left(x^{\nu+1}\right) .
\end{aligned}
$$

Hence, for sufficiently small $a$ we obtain

$$
\begin{aligned}
\theta(a, \lambda) & \cot \beta+D_{q} \theta(a, \lambda) \\
= & -c_{1} c_{2} \frac{\left(q^{2 \nu+2} ; q^{2}\right)_{\infty}}{\left(q^{2} ; q^{2}\right)_{\infty}} q^{-\frac{1}{2}} s^{\nu} J_{-\nu}\left(b s q^{-\nu / 2}\right) \\
& \times\left(a^{\nu+\frac{1}{2}} \cot \beta+\left(\frac{1}{1+\sqrt{q}}+\frac{1-q^{\nu}}{1-q} q^{\frac{1}{2}}\right) a^{\nu-\frac{1}{2}}\right)
\end{aligned}
$$




$$
\begin{aligned}
& +c_{1} c_{2} \frac{\left(q^{-2 \nu+2} ; q^{2}\right)_{\infty}}{\left(q^{2} ; q^{2}\right)_{\infty}} q^{-\frac{1}{2}} s^{-\nu} q^{\nu^{2} / 2} J_{\nu}(b s) \\
& \times\left(a^{-\nu+\frac{1}{2}} \cot \beta+\left(\frac{1}{1+\sqrt{q}}+\frac{1-q^{-\nu}}{1-q} q^{\frac{1}{2}}\right) a^{-\nu-\frac{1}{2}}\right) \\
& +O\left(a^{-\nu+\frac{1}{2}}\right)
\end{aligned}
$$

and

$$
\begin{aligned}
\phi(a, \lambda) & \cot \beta+D_{q} \phi(a, \lambda)-\frac{1}{b \sqrt{1+q}}\left(\theta(a, \lambda) \cot \beta-D_{q} \theta(a, \lambda)\right) \\
= & -c_{1} c_{2} \frac{\left(q^{2 \nu+2} ; q^{2}\right)_{\infty}}{\left(q^{2} ; q^{2}\right)_{\infty}} q^{-\frac{1}{2}} s^{\nu+1} D_{q} J_{-\nu}\left(b s q^{-\nu / 2}\right) \\
& \times\left(a^{\nu+\frac{1}{2}} \cot \beta+\left(\frac{1}{1+\sqrt{q}}+\frac{1-q^{\nu}}{1-q} q^{\frac{1}{2}}\right) a^{\nu-\frac{1}{2}}\right) \\
& +c_{1} c_{2} \frac{\left(q^{-2 \nu+2} ; q^{2}\right)_{\infty}}{\left(q^{2} ; q^{2}\right)_{\infty}} q^{-\frac{1}{2}} s^{-\nu+1} q^{\nu^{2} / 2} D_{q} J_{\nu}(b s) \\
& \times\left(a^{-\nu+\frac{1}{2}} \cot \beta+\left(\frac{1}{1+\sqrt{q}}+\frac{1-q^{-\nu}}{1-q}\right) a^{-\nu-\frac{1}{2}} q^{\frac{1}{2}}\right) \\
& +O\left(a^{-\nu+3 / 2}\right) .
\end{aligned}
$$

Now let

$$
\begin{aligned}
& \frac{\left(a^{\nu+\frac{1}{2}} \cot \beta+\left(\frac{1}{1+\sqrt{q}}+\frac{1-q^{\nu}}{1-q} q^{\frac{1}{2}}\right) a^{\nu-\frac{1}{2}}\right)}{\left(q^{-2 \nu+2} ; q^{2}\right)_{\infty}} \\
& \quad=c \frac{\left(a^{-\nu+\frac{1}{2}} \cot \beta+\left(\frac{1}{1+\sqrt{q}}+\frac{1-q^{-\nu}}{1-q} q^{\frac{1}{2}}\right) a^{-\nu-\frac{1}{2}}\right)}{\left(q^{2 \nu+2} ; q^{2}\right)_{\infty}},
\end{aligned}
$$

where $c$ is a constant. Then

$$
\begin{aligned}
\cot \beta & =\frac{c\left(q^{-2 \nu+2} ; q^{2}\right)_{\infty}\left(\frac{1}{1+\sqrt{q}}+\frac{1-q^{-\nu}}{1-q} q^{\frac{1}{2}}\right) a^{-\nu-\frac{1}{2}}-\left(q^{2 \nu+2} ; q^{2}\right)_{\infty}\left(\frac{1}{1+\sqrt{q}}+\frac{1-q^{\nu}}{1-q}\right) a^{\nu-\frac{1}{2}}}{\left(q^{2 \nu+2} ; q^{2}\right)_{\infty} a^{\nu+\frac{1}{2}}-c\left(q^{-2 \nu+2} ; q^{2}\right)_{\infty} a^{-\nu+\frac{1}{2}}} \\
& =O\left(\frac{1}{a}\right)
\end{aligned}
$$

as $a \rightarrow 0$ and

$$
a^{\nu+\frac{1}{2}} \cot \beta+\left(\frac{1}{1+\sqrt{q}}+\frac{1-q^{\nu}}{1-q} q^{\frac{1}{2}}\right) a^{\nu-\frac{1}{2}}=O\left(a^{\nu-\frac{1}{2}}\right) .
$$


Hence, the O-terms in equations (6.4) and (6.5) are negligible if $3 / 2-\nu>$ $\nu-1 / 2$, that is, $\nu<1$. Consequently, as $a \rightarrow 0$,

$$
\begin{aligned}
m(\lambda)= & \lim _{a \rightarrow 0} l_{a}(\lambda) \\
= & -s \frac{q^{\nu^{2} / 2} s^{-\nu} D_{q} J_{\nu}(b s)-c s^{\nu} D_{q} J_{-\nu}\left(b s q^{-\nu / 2}\right)}{q^{\nu^{2} / 2} s^{-\nu} J_{\nu}(b s)-c s^{\nu} J_{-\nu}\left(b s q^{-\nu / 2}\right)} \\
& -\frac{1}{(1+\sqrt{q}) b} .
\end{aligned}
$$

Since $c$ may have any value, $m$ describes the circles obtained by varying $c$, and so this is the limit-circle. For each value of $c, m$ is an even function of $s$ and so is a one-valued function of $\lambda$. Its only singularities are poles which are the zeros of the function $s^{-\nu} J_{\nu}(b s)-c s^{\nu} J_{-\nu}\left(b s q^{-\nu / 2}\right)$, which by using the same technique of [31] can be shown to be countable, real, and simple. Denoting these by $\lambda_{n}$, we have

$$
\begin{aligned}
& \theta\left(x, \lambda_{n}\right)=-\frac{q^{-\nu(\nu-1)-\frac{1}{2}} \sqrt{b}}{1+q} \Gamma_{q^{2}}(\nu) \Gamma_{q}^{2}(1-\nu) \\
& \times x^{\frac{1}{2}}\left\{J_{\nu}\left(x \sqrt{\lambda_{n}}\right) J_{-\nu}\left(b q^{-\nu / 2} \sqrt{\lambda_{n}}\right)-J_{-\nu}\left(x q^{-\nu / 2} \sqrt{\lambda_{n}}\right) J_{\nu}\left(b \sqrt{\lambda_{n}}\right)\right\} \\
& =-\frac{q^{-\nu(\nu-1)-\frac{1}{2}} \sqrt{b}}{1+q} \Gamma_{q^{2}}(\nu) \Gamma_{q}^{2}(1-\nu) x^{\frac{1}{2}} J_{\nu}\left(b \sqrt{\lambda_{n}}\right) \\
& \times\left\{c^{-1} \lambda_{n}^{-\nu} J_{\nu}\left(x \sqrt{\lambda_{n}}\right)-J_{-\nu}\left(x q^{-\nu / 2} \sqrt{\lambda_{n}}\right)\right\} .
\end{aligned}
$$

The normalized eigenfunctions are $\left|r_{n}\right|^{1 / 2} \theta\left(x, \lambda_{n}\right)$, where $r_{n}$ is the residue of $m(\lambda)$ at the point $\lambda_{n}$. For $c=\infty$ the expansion is the ordinary Fourier expansion of order $-\nu$, and for $c=0$ it is the expansion of order $-\nu$. In the case when $\nu=0$, we have (see [45])

$$
\begin{aligned}
Y_{0}(x ; q)=\frac{-(1-q)}{\pi(q ; q)_{\infty} \log q}( & (q ; q)_{\infty}\left(\frac{1}{2} \log q+2 \log x\right) J_{0}(x ; q) \\
& +2 \sum_{k=0}^{\infty}(-1)^{k} q^{k(k+1) / 2} F^{\prime}(k+1) \frac{x^{2 k}}{(q ; q)_{k}} \\
& \left.+(q ; q)_{\infty} \log q \sum_{n=0}^{\infty}(-1)^{n} n \frac{q^{n(n+1) / 2}}{(q ; q)_{n}^{2}} x^{2 n}\right)
\end{aligned}
$$


where $F(t):=\left(q^{t} ; q\right)_{\infty}, t \in \mathbb{C}$. Then for each fixed $s$, we have

$$
\begin{aligned}
Y_{0}(s x) & =\frac{\left(1-q^{2}\right)}{2 \pi \log q}(\gamma+\log (x s))\left\{1+O\left(x^{2}\right)\right\}, \\
D_{q} Y_{0}(s x) & =\frac{2}{\pi\left(q^{2} ; q^{2}\right)_{\infty} x s}+O(x|\log x|)
\end{aligned}
$$

as $x \rightarrow 0$, where $\gamma=\log q-2 /\left(\pi\left(q^{2} ; q^{2}\right)_{\infty}\right)\left(\Gamma_{q}^{\prime}(1)+\log \left(1-q^{2}\right)\right)$. Hence,

$$
\begin{aligned}
\phi(a, \lambda) \cot \beta+D_{q} \phi(a, \lambda)-\frac{1}{b(1+\sqrt{q})}\left(\theta(a, \lambda) \cot \beta-D_{q} \theta(a, \lambda)\right) \\
=c_{1} s a^{-\frac{1}{2}}\left(a \cot \beta+\frac{1}{1+\sqrt{q}}\right)\left(D_{q} Y_{0}(b s)-\frac{\left(1-q^{2}\right) \log s}{2 \pi \log q} J_{0}(b s)\right) \\
\quad-c_{1} s a^{-\frac{1}{2}} J_{0}(b s)\left(\left(a \cot \beta+\frac{1}{1+\sqrt{q}}\right) \frac{\left(1-q^{2}\right) \log a \gamma}{2 \pi \log q}+\frac{2 q^{\frac{1}{2}}}{\pi\left(q^{2} ; q^{2}\right)_{\infty} s}\right) \\
+O\left(a^{3 / 2}|\log a|\right) .
\end{aligned}
$$

Taking

$$
\left(a \cot \beta+\frac{1}{1+\sqrt{q}}\right) \frac{\left(1-q^{2}\right) \log a \gamma}{2 \pi \log q}+\frac{2 q^{\frac{1}{2}}}{\pi\left(q^{2} ; q^{2}\right)_{\infty} s}=c\left(a \cot \beta+\frac{1}{1+\sqrt{q}}\right)
$$

and proceeding as before, we obtain

$$
\begin{aligned}
m(\lambda)= & -q^{\frac{1}{2}} s \frac{c D_{q} J_{0}(b s)-\left\{D_{q} Y_{0}-\frac{\left(1-q^{2}\right)}{2 \pi \log q}(\gamma+\log s) D_{q} J_{0}(b s)\right\}}{c J_{0}(b s)-\left\{Y_{0}-\frac{\left(1-q^{2}\right)}{2 \pi \log q}(\gamma+\log s) J_{0}(b s)\right\}} \\
& -\frac{1}{(1+\sqrt{q}) b} .
\end{aligned}
$$

From (6.6), $m(\lambda)$ is an even function of $s$ and is a single-valued function of $\lambda$. Its only singularities are poles at the zeros of the function

$$
c J_{0}(b s)-\left\{Y_{0}-\frac{\left(1-q^{2}\right)}{2 \pi \log q}(\gamma+\log s) J_{0}(b s)\right\} .
$$

Denoting these zeros by $\mu_{n}, n \in \mathbb{Z}^{+}$, we have

$$
\begin{aligned}
& \theta\left(x, \mu_{n}\right) \\
& \quad=c_{1} q^{-\frac{1}{2}} x^{\frac{1}{2}}\left\{J_{0}\left(x \sqrt{\mu_{n}}\right) Y_{0}\left(b \sqrt{\mu_{n}}\right)-Y_{0}\left(x \sqrt{\mu_{n}}\right) J_{0}\left(b \sqrt{\mu_{n}}\right)\right\} \\
& =c_{1} q^{-\frac{1}{2}} x^{\frac{1}{2}} J_{0}(b s)\left\{\left(\frac{\left(1-q^{2}\right)}{2 \pi \log q}(\gamma+\log s)+c\right) J_{0}\left(x \sqrt{\mu_{n}}\right)-Y_{0}\left(x \sqrt{\mu_{n}}\right)\right\} .
\end{aligned}
$$


The normalized eigenfunctions are $\left|r_{n}\right|^{1 / 2} \theta\left(x, \sqrt{\mu_{n}}\right)$, where the $r_{n}$ are the residue of $m(\lambda)$ at the points $\mu_{n}$.

\section{REFERENCES}

[1] L. D. Abreu, A q-sampling theorem related to the q-Hankel transform, Proc. Amer. Math. Soc. 133 (2004), 1197-1203.

[2] - Functions q-orthogonal with respect to their own zeros, Proc. Amer. Math. Soc. 134 (2006), 2695-2701.

[3] L. D. Abreu and J. Bustoz, "On the completeness of sets of $q$-Bessel functions" in Theory and Applications of Special Functions, Dev. Math. 13, Springer, New York, 2005, 29-38.

[4] L. D. Abreu, J. Bustoz, and J. L. Caradoso, The roots of the third Jackson q-Bessel functions, Int. J. Math. Math. Sci. 67 (2003), 4241-4248.

[5] M. H. Abu-Risha, M. H. Annaby, M. E. H. Ismail, and Z. S. Mansour, Linear qdifference equations, Z. Anal. Anwend. 26 (2007), 481-494.

[6] L. Ahlfors, Complex Analysis, Cambridge University Press, Cambridge, 1999.

[7] G. E. Andrews, R. Askey, and R. Roy, Special Functions, Cambridge University Press, Cambridge, 1999.

[8] M. H. Annaby, q-Type sampling theorems, Results Math. 44 (2003), 214-225.

[9] M. H. Annaby and Z. S. Mansour, Basic Sturm-Liouville problems, J. Phys. A 38 (2005), 3775-3797; Correction, J. Phys. A 39 (2006), 8747.

[10] (2006), 1091-1103.

[11] - On the zeros of the second and third Jackson q-Bessel functions and their associated q-Hankel transforms, Math. Proc. Cambridge Philos. Soc. 147 (2009), $47-67$.

[12] - Asymptotic formulae for eigenvalues and eigenfunctions of $q$-Sturm-Liouville problems, Math. Nachr. 284 (2011), 443-470.

[13] J. M. Berezanskii, Expansions in Eigenfunctions of Selfadjoint Operators, Amer. Math. Soc., Providence, 1968.

[14] B. M. Brown, J. S. Christiansen, and K. M. Schmidt, Spectral properties of a $q$ Sturm-Liouville operator, Comm. Math. Phys. 287 (2009), 259-274.

[15] B. M. Brown, W. D. Evans, and M. E. H. Ismail, The Askey-Wilson polynomials and q-Sturm-Liouville problems, Math. Proc. Cambridge Philos. Soc. 119 (1996), 1-16.

[16] J. Bustoz and J. L. Cardoso, Basic analog of Fourier series on a q-linear grid, J. Approx. Theory 112 (2001), 134-157.

[17] J. Bustoz and S. K. Suslov, Basic analog of Fourier series on a q-quadratic grid, Methods Appl. Anal. 5 (1998), 1-38.

[18] R. D. Carmichael, The general theory of linear q-difference equations, Amer. J. Math. 34 (1912), 147-168.

[19] - Linear difference equations and their analytic solutions, Trans. Amer. Math. Soc. 12, no. 1 (1911), 99-134.

[20] - On the theory of linear difference equations, Amer. J. Math. 35 (1913), $163-182$.

[21] J. S. Christiansen and M. E. H. Ismail, A moment problem and a family of integral evaluations, Trans. Amer. Math. Soc. 358, no. 9 (2006), 4071-4097.

[22] R. Conway, Functions in One Complex Variable, Springer, New York, 1999. 
[23] C. T. Fulton, Parametrizations of Titchmarsh's $m(\lambda)$-functions in the limit circle case, Trans. Amer. Math. Soc. 229 (1977), 51-63.

[24] W. Hahn, Beiträge zur Theorie der Heineschen Reihen, Math. Nachr. 2 (1949), 340379 .

[25] M. Hajmirzaahmad and A. M. Krall, Singular second-order operators: the maximal and minimal operators, and selfadjoint operators in between, SIAM Rev. 34 (1992), 614-634.

[26] M. E. H. Ismail, The zeros of basic Bessel functions, the functions $J_{\nu+a x}(x)$ and associated orthogonal polynomials, J. Math. Anal. Appl. 86 (1982), 1-19.

[27] — Classical and Quantum Orthogonal Polynomials in One Variable, Encyclopedia Math. Appl. 98, Cambridge University Press, Cambridge, 2005.

[28] - On Jackson's third q-Bessel function, preprint, 1996.

[29] F. H. Jackson, The applications of basic numbers to Bessel's and Legendre's equations, Proc. Lond. Math. Soc. (3) 2 (1905), 192-220.

[30] - On q-definite integrals, Quart. J. Pure Appl. Math. 41 (1910), 193-203.

[31] H. T. Koelink and R. F. Swarttouw, On the zeros of the Hahn-Exton q-Bessel function and associated q-Lommel polynomials, J. Math. Anal. Appl. 186 (1994), 690-710.

[32] T. H. Koornwinder and R. F. Swarttouw, On a q-analog of the Fourier and Hankel transforms, Trans. Amer. Math. Soc. 333 (1992), no. 1, 445-461.

[33] S. Lang, Complex Analysis, Springer, New York, 1992.

[34] A. Lavagno, Basic-deformed quantum mechanics, Rep. Math. Phys. 64 (2009), 79-91.

[35] N. Levinson, A simplified proof of the expansion theorem for singular second order linear differential equations, Duke Math. J. 18 (1951), 57-71.

[36] B. M. Levitan and I. S. Sargsjan, Introduction to Spectral Theory: Selfadjoint Ordinary Differential Operators, Amer. Math. Soc., Providence, 1975.

[37] - Sturm Liouville and Dirac Operators, Kluwer, Dordrecht, 1991.

[38] A. Matsuo, Jackson integrals of Jordan-Pochhammer type and quantum KnizhnikZamolodchikov equations, Comm. Math. Phys. 151 (1993), 263-273.

[39] M. A. Naimark, Linear Differential Operators, Part II: Lineal Differential Operators in Hilbert Space, Frederick Ungar, New York, 1968.

[40] M. H. Stone, A comparison of the series of Fourier and Birkhoff, Trans. Amer. Math. Soc. 28 (1926), 695-761.

[41] - Linear Transformations in Hilbert Space and Their Application to Analysis, Amer. Math. Soc., Providence, 1932.

[42] S. K. Suslov, Some expansions in basic Fourier series and related topics, J. Approx. Theory 115 (2002), 289-353.

[43] — An Introduction to Basic Fourier Series, Kluwer Ser. Dev. Math. 9, Kluwer Academic, Dordrecht, 2003.

[44] R. F. Swarttouw, The Hahn-Exton q-Bessel function, Ph.D. dissertation, Technical University of Delft, Delft, Netherlands, 1992.

[45] R. F. Swarttouw and H. G. Meijer, A q-analogue of the Wronskian and a second solution of the Hahn-Exton q-Bessel difference equation, Proc. Amer. Math. Soc. 120 (1994), 855-864.

[46] E. C. Titchmarsh, On the uniqueness of the Green's function associated with a secondorder differential equation, Canad. J. Math. 1 (1949), 191-198.

[47] — Eigenfunction Expansions Associated with Second-Order Differential Equations, I, 2nd ed., Clarendon Press, Oxford, 1962. 
[48] H. Weyl, Gewöhnliche linear differentialgleichungen mit singularitäten stellen und ihre eigenfunktionen, Göttingen Ges. Wiss. Nach. 68 (1909), 73-64.

[49] - Gewöhnliche linear differentialgleichungen mit singularitäten stellen und ihre eigenfunktionen, Göttingen Ges. Wiss. Nach. 68 (1910), 442-467.

[50] —, Über Gewöhnliche differentialgleichungen mit singularitäten und die zugehörigen entwicklung willkürlicher funktionen, Math. Ann. 68 (1910), 220-269.

[51] K. Yosida, On Titchmarsh-Kodaira's formula concerning Weyl-Stone's eingenfunction expansion, Nagoya Math. J. 1 (1950), 49-58.

[52] - Lectures on Differential and Integral Equations, Springer, New York, 1960.

M. H. Annaby

Department of Mathematics, and statistics and Physics

Qatar University

Doha

Qatar

mannaby@qu.edu.qa

Z. S. Mansour

Department of Mathematics

King Saudi University, Riyadh

Riyadh 11451

Kingdom of Saudi Arabia

zeinabs98@hotmail.com

I. A. Soliman

Department of Mathematics

Cairo University

Giza

Egypt

eman.a.raouf@hotmail.com 